\title{
MECHANISTIC INVESTIGATIONS OF THE HYDROLYSIS OF AMIDES, OXOESTERS AND THIOESTERS VIA KINETIC ISOTOPE EFFECTS AND POSITIONAL ISOTOPE EXCHANGE
}

\author{
Lori I. Robins ${ }^{\mathrm{a}}$, Emily J. Fogle ${ }^{\mathrm{b}}$ and John F. Marlier ${ }^{\mathrm{b} *}$ \\ ${ }^{a}$ Department of Biochemistry, University of Washington-Bothell, Bothell, WA 98011, \\ USA \\ ${ }^{\mathrm{b}}$ Department of Chemistry and Biochemistry, California Polytechnic State University, \\ San Luis Obispo, CA 93407 USA
}

Keywords:

Amide hydrolysis mechanisms

Oxoester hydrolysis mechanisms

Thioester hydrolysis mechanisms

Kinetic isotope effects

Positional isotope exchange

${ }^{a}$ Abbreviations: KIE, Kinetic Isotope Effect; EIE, Equilibrium Isotope Effect; TIF, Temperature Independent Factor; TDF, Temperature Dependent Factor; FTC, Formylthiocholine; BChE, Butyrylcholinesterase.

*Corresponding author at Department of Chemistry and Biochemistry, California Polytechnic State University, 1 Grand Avenue, San Luis Obispo, CA 93407 USA. Phone: (805) 756-1327. E-mail: jmarlier@,calpoly.edu

\begin{abstract}
The hydrolysis of amides, oxoesters and thioesters is an important reaction in both organic chemistry and biochemistry. Kinetic isotope effects (KIEs) are one of the most important physical organic methods for determining the most likely transition state structure and rate-determining step of these reaction mechanisms. This method induces a very small change in reaction rates, which, in turn, results in a minimum disturbance of the natural mechanism. KIE studies were carried out on both the non-enzymatic and the enzyme-catalyzed reactions in an effort to compare both types of mechanisms. In these studies the amides and esters of formic acid were chosen because this molecular structure allowed development of methodology to determine heavy-atom solvent (nucleophile) KIEs. This type of isotope effect is difficult to measure, but is rich in mechanistic information. Results of these investigations point to transition states with varying degrees of tetrahedral character that fit a classical stepwise mechanism. This article is part of a special issue entitled: Enzyme Transition States from Theory and Experiment.
\end{abstract}




\subsection{The chemical and biochemical significance of acyl group hydrolysis and limitations of this review}

There are several types of acyl groups; those of prime importance in biology are amides, oxoesters, thioesters and anhydrides. The term oxoester is used throughout this review to differentiate the esters with oxygen from those with sulfur leaving groups. In biology the roles of these functional groups are diverse. For example, the amide group forms the backbone of proteins, esters are an integral part of lipid metabolism, thioesters serve as acyl donors and anhydrides have a well-known role in the transfer of energy and in the transfer of functional groups. As a result, the non-enzymatic and enzyme-catalyzed reactions of these functional groups have been the subject of mechanistic studies for many decades $[1,2,3,4,5]$. To accommodate the truly vast volume of literature this review will be limited to: (a) results of KIE studies on the hydrolysis reaction of amides, oxoesters and thioesters, (b) a qualitative discussion (as opposed to theory-based calculations) of the results of these KIE studies and, (c) a focus on the formyl-H, the leaving group, and the solvent nucleophile-O KIEs. Although the carbonyl-C and carbonyl-O KIEs are listed for completeness, they have generally been of less importance in proposing transition state structures; several reasons for this are stated in section 5.5.

\subsection{Brief kinetic isotope effect methodology and terminology}

In the simplest terms KIEs reflect bonding changes between the ground state of the reactant and the transition state of highest energy. The physical origin of KIEs is a complex subject and the theory has advanced steadily over the years $[6,7,8,9]$. This topic will be discussed elsewhere in this special issue.

There are two general methods for measuring KIEs [3]. The first is the direct method in which the rate constants for molecules containing the heavier and lighter isotopes are measured independently. The KIE was formerly reported as the ratio of rate constants for the molecule containing the lighter isotope over that for the heavier one. For example, an oxygen KIE would be reported as ${ }^{16} k /{ }^{18} k$. In more modern nomenclature the KIE is simply stated as heavy $k$ (e.g. ${ }^{18} k$ ) [10]. The direct method is the method of choice for most hydrogen KIEs due to the large magnitude of these effects. However, for atoms heavier than hydrogen the difference between the reaction rates for the two isotopic species is much smaller (on the order of a few percent). In this case the competitive or indirect method is generally used. For the hydrolysis reactions discussed herein, the competitive method specifically involved allowing a natural abundance mixture of the reactant to compete for the solvent nucleophile within the same solution. This method requires precise measurement of the isotope ratios (heavy/light) of: (a) the reactant or the product at some known fraction of reaction and (b) the reactant prior to commencement of the reaction or the product after complete conversion. Our isotope ratios were measured on an isotope ratio mass spectrometer (IRMS), which has the required precision to determine these small magnitude KIEs. The IRMS compares the isotope ratio of the sample ( $\left.\mathrm{R}_{\text {sample }}\right)$ to that of a tank standard ( $\left.\mathrm{R}_{\text {standard }}\right)$. The actual isotope ratio (heavy/light) of a particular atom is generally not what is actually published. Instead the modern IRMS gives isotopic composition in delta $(\delta)$ notation, which is the per mil difference between the isotope ratio of a sample and that for a standard. The relationship between an isotope ratio and its corresponding delta is given in eq 1 . In delta notation a superscript denotes the isotopes in question (e.g. ${ }^{13} \delta$ for the ${ }^{13} \mathrm{C} /{ }^{12} \mathrm{C}$ ratio; ${ }^{34} \delta$ is for the 
${ }^{34} \mathrm{~S} /{ }^{32} \mathrm{~S}$ ratio). Published equations then allow calculation of the KIE from these measured delta values [3]. A negative value of $\delta$ means the sample is lighter (less of the heavy isotope) than the standard; a positive value means the opposite. In enzyme-catalyzed reactions the competitive method gives an isotope effect on $\mathrm{V} / \mathrm{K}$. These are reported as ${ }^{\text {heavy }}(V / K)$ or, for example, as ${ }^{18}(V / K)$ for an ${ }^{18}$ O KIE.

$$
\delta=\left(\left(\mathrm{R}_{\text {sample }} / \mathrm{R}_{\text {standard }}\right)-1\right) * 1000
$$

\subsection{Positional isotope exchange (PIX)}

PIX experiments have been used for more than five decades to offer evidence for the existence of tetrahedral intermediates in the stepwise mechanism [5,11]. This experiment compares the overall rate of hydrolysis $\left(k_{\mathrm{h}}\right)$ to the rate of ${ }^{18} \mathrm{O}$ exchange $\left(k_{\mathrm{ex}}\right)$ between water and the carbonyl-O of the substrate or product. The results are reported as the ratio $k_{\mathrm{h}} / k_{\mathrm{ex}}$. The experimental protocol has several variations. One can measure the rate of exchange from ${ }^{18} \mathrm{O}$-water into the carbonyl-O of substrate or product (assuming the product does not independently exchange ${ }^{18} \mathrm{O}$ with water). Similarly one can measure the rate of loss of ${ }^{18} \mathrm{O}$ from the carbonyl-O of labeled substrate into water.

Interpretation of the resulting $k_{\mathrm{h}} / k_{\mathrm{ex}}$ ratios is not straightforward. Normally the oxygen atoms of the tetrahedral intermediates have differing number of protons. In order to convert $k_{\mathrm{h}} / k_{\text {ex }}$ into a partition ratio for formation and breakdown of the tetrahedral intermediate one must assume that proton transfers are much faster than any other mechanistic steps. This means tetrahedral intermediates are at protonic equilibria. This reasonable assumption is commonly used in hydrolysis reactions [5]. The reader should be aware that we utilize this simplifying assumption in the discussions that follow.

\subsection{Proposed hydrolysis mechanisms}

Three general mechanisms have been proposed for the non-enzymatic hydrolysis of amides, oxoesters and thioesters $[12,13,14]$. The mechanisms given below are for alkaline hydrolysis. The proposed mechanisms differ somewhat under acidic and neutral conditions, most notably in the charge of the intermediates. The most common mechanism encountered during hydrolysis is the stepwise mechanism (Scheme 1, top). There is reasonable evidence for the concerted mechanism (Scheme 1, middle) in the case of oxoesters with exceptional leaving groups, such as those containing $p$-nitrophenyl [15]. There is evidence for the dissociative mechanism (Scheme 1, bottom) in the case of acyl chloride solvolysis [16]. 


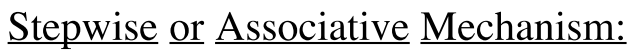

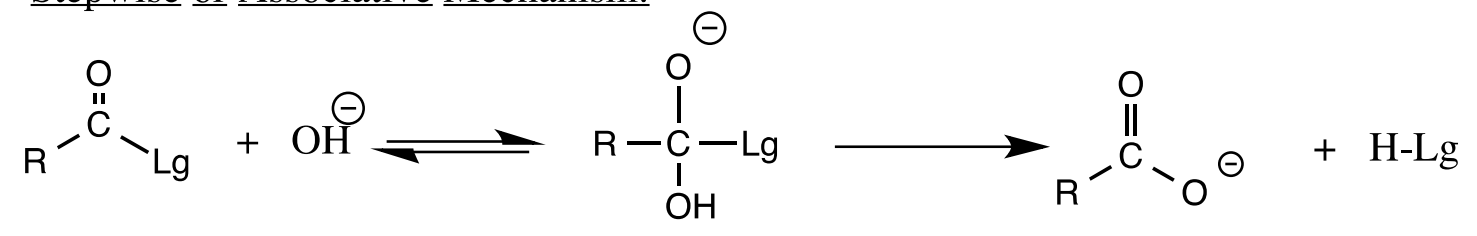

Concerted Mechanism:

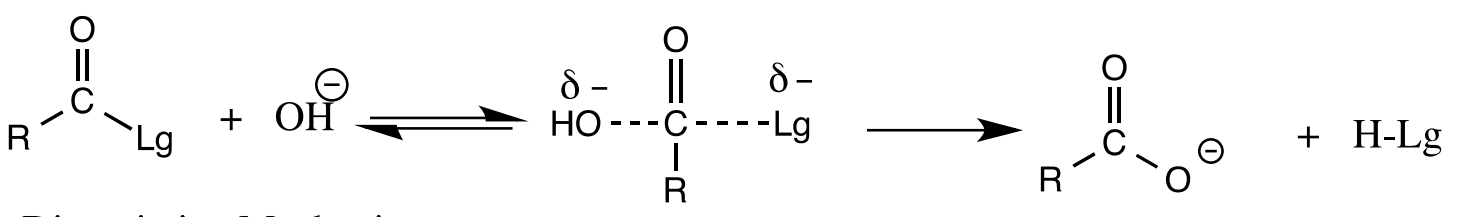

Dissociative Mechanism:<smiles>[2H]C(=O)[Hg]</smiles>

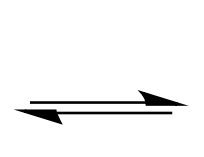

$\oplus \underset{\substack{l \\ \mathrm{R}}}{\mathrm{O}}+\mathrm{Lg}^{\ominus}$<smiles>O=C(O)CC(=O)O</smiles>

Scheme 1. Possible mechanisms for acyl group hydrolysis. (Note: only alkaline hydrolysis is shown here.)

Some controversy exists concerning the stability of fully charged tetrahedral intermediates in aqueous media [17]. It has been proposed that some tetrahedral intermediates do not have a sufficiently long lifetime to be considered as a true intermediate. In this argument the actual charged species actually share the positive or negative charge on heteroatoms like oxygen with a chain of several water molecules. Although this review will show traditional structures with the charges localized, the reader should be aware that this issue is subject to ongoing investigation.

\subsection{The Alkaline hydrolysis of formamide}

Early kinetic experiments on alkyl amides established that hydrolysis was firstorder in $\mathrm{OH}^{-}$at low base concentrations. However, at higher base concentrations the rate became second-order in $\mathrm{OH}^{-}$. This result suggested a change in mechanism with $\mathrm{pH}$ $[18,19]$, as is shown in Scheme 2. In this mechanism $\mathrm{OH}^{-}$is more accurately represented as a hydrate, $\left(\mathrm{H}_{2} \mathrm{O}\right)_{n} \mathrm{OH}^{-}$; the reason for this will be clarified later in this discussion. However, to avoid over-complication of mathematical equations (such as eq 2), the simpler term, $\left[\mathrm{OH}^{-}\right]$is used to represent $\left(\mathrm{H}_{2} \mathrm{O}\right)_{n} \mathrm{OH}^{-}$. 


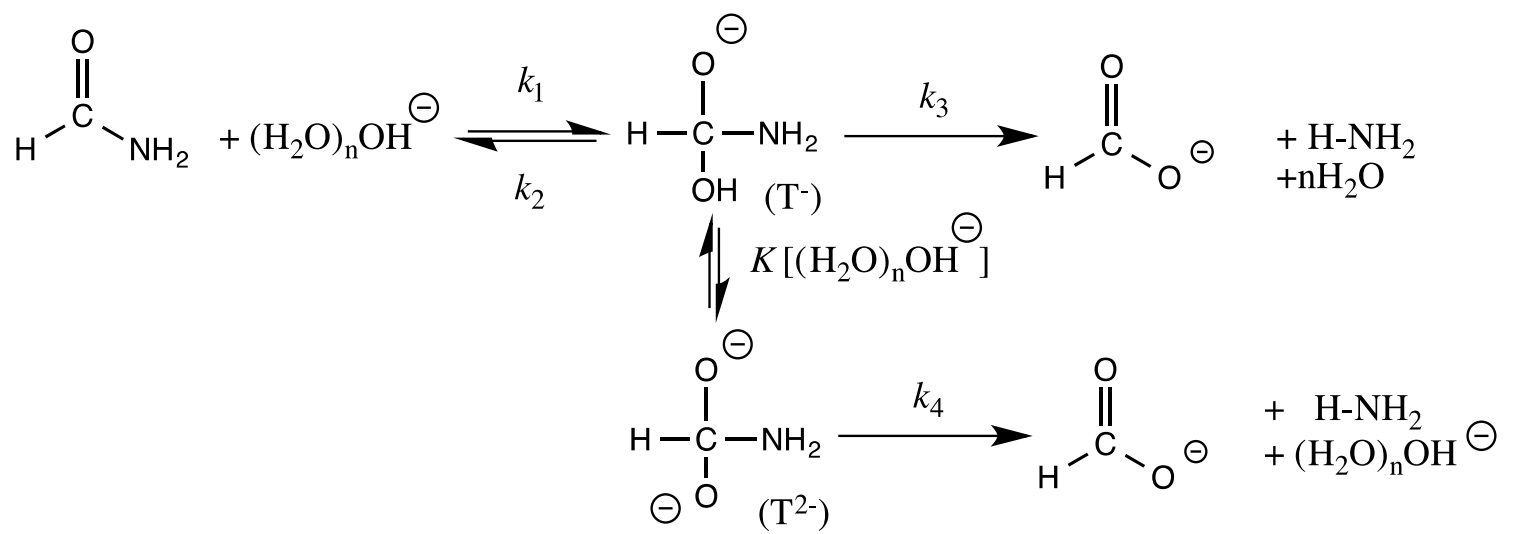

Scheme 2. Proposed mechanism for alkaline hydrolysis of formamide.

PIX experiments with formamide are consistent with this mechanism, where $k_{\mathrm{h}} / k_{\text {ex }}$ was found to vary from 2.5 at $0.092 \mathrm{M} \mathrm{OH}^{-}$to 8.3 at $1.46 \mathrm{M} \mathrm{OH}^{-}$[20]. A plot of $k_{\mathrm{h}} / k_{\mathrm{ex}}$ versus $\left[\mathrm{OH}^{-}\right]$closely fit eq. 2 , which is derived from the steady-state rate laws for hydrolysis and for exchange from the mechanism in Scheme 2 [5]. This plot allowed calculation of the partition ratios $\left(K k_{4} / k_{2}=2.10\right)$ and $k_{3} / k_{2}=1.05$.

$$
k_{h} / k_{e x}=\left(2 k_{3}+2 k_{4} K O H\right) / k_{2}
$$

The above kinetic and PIX experiments resulted in a viable mechanism for which reasonable intermediates are proposed. Our multiple KIE study of the alkaline hydrolysis goes one step further; it allows postulation of bond order in the transition state [20]. The results of the KIE experiments are summarized in Fig. 1A. In order to obtain the clearest transition state structure, our experimental design included measuring KIEs for as many atoms at the reactive center of the substrate as was experimentally possible. It must be noted that our KIEs were measured at low concentrations of $\mathrm{OH}^{-}(0.09-0.20 \mathrm{M})$ and the top pathway in Scheme 2 is the dominant one. As a simplifying approximation the bottom pathway and the $K k_{4} / k_{2}$ term is ignored. This makes $k_{3} / k_{2}=1.05$ a good estimate of the partition ratio of $\mathrm{T}^{-}$.

A. Alkaline Hydrolysis:

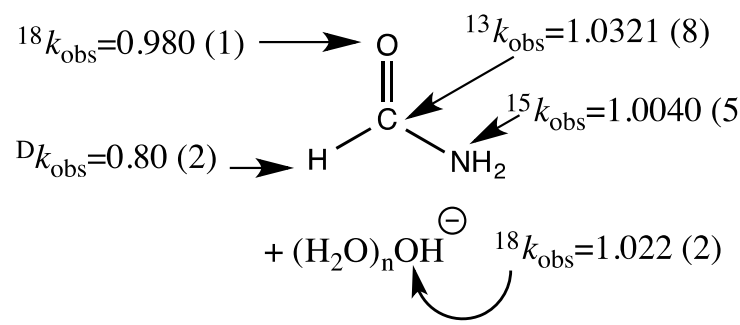

B. Acid-Catalyzed Hydrolysis:

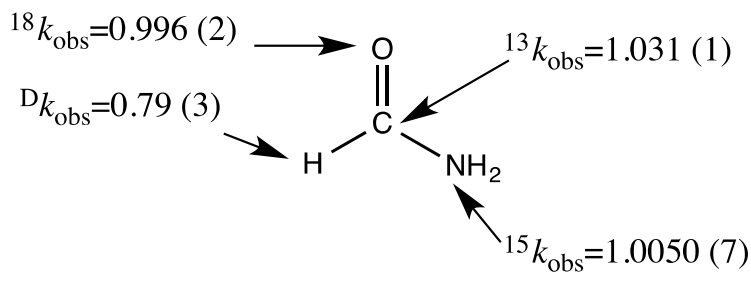

Fig. 1. KIE Summary for the Alkaline (A) and Acid-Catalyzed (B) Hydrolysis of Formamide. Numbers in parentheses are the standard error in the right-most decimal place. 
Three of the five measured KIEs are of prime importance in estimating the extent of bonding changes on going to the transition state: the formyl- $\mathrm{H}$, the leaving- $\mathrm{N}$, and the nucleophile-O. The formyl-H KIE is a secondary KIE because the $\mathrm{H}-\mathrm{C}$ bond is not broken during the reaction; it is sensitive to changes in hybridization at the carbonyl-C on going to the transition state. It is known that going from an $\mathrm{sp}^{2}$ ground state to an $\mathrm{sp}^{3}$-like transition state will give an inverse KIE, whereas the opposite transformation $\left(\mathrm{sp}^{3} \rightarrow \mathrm{sp}^{2}\right)$ will show a normal KIE [21]. In the present case interpretation of the formyl-H KIE is more complex because the ratio $k_{3} / k_{2}$ is only 1.05 , indicating that the transition states for formation and breakdown of $\mathrm{T}^{-}$would be of comparable energy. As a result both transition states must be considered in explaining the observed KIE results. Qualitatively, it is expected that the transition state for formation of $\mathrm{T}^{-}$(the $k_{1}$ step) should contain an increasing amount of $\mathrm{sp}^{3}$ character and lead to an inverse KIE as seen in Fig. 2, Structure I. For equations and transition state structures in this review (IIIIII) represents hydrogen bonds and (----) represents bonds in transition.

On the other hand, the transition state for the decomposition of $\mathrm{T}^{-}$(the $k_{3}$ step) should show a normal KIE since the change is $\mathrm{sp}^{3} \rightarrow \mathrm{sp}^{2}$ (Fig. 2, Structure II). The observed formyl-H KIE can be reconciled with both transition states via an estimation of the intrinsic KIEs for the $k_{1}$ and $k_{3}$ steps in the mechanism. This estimation involves the use of model equilibria and the partition ratio $\left(k_{3} / k_{2}\right)$ given above (see reference 20 for detailed calculations). These calculations yield intrinsic formyl-H KIEs of ${ }^{\mathrm{D}} k_{1}=0.69$ and of ${ }^{\mathrm{D}} k_{3}=1.34$.
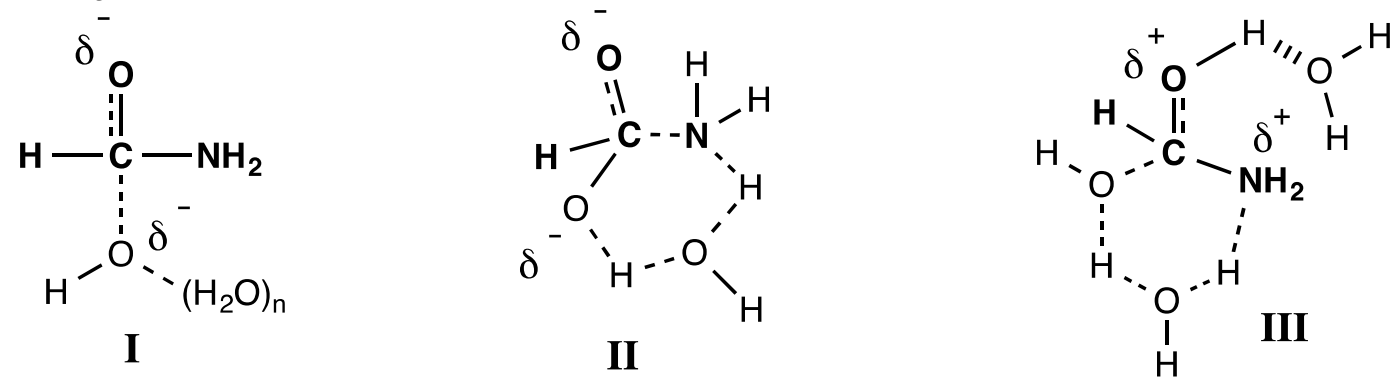

Fig. 2. Possible transition state structures for alkaline (I, II) and acidic (III) hydrolysis of formamide.

The magnitude of the leaving-N KIE is small $\left({ }^{15} k_{\mathrm{obs}}=1.004\right)$ initially indicating a small amount of $\mathrm{C}-\mathrm{N}$ bond cleavage. For reference a large primary nitrogen KIE for reactions in solution can be as high as ${ }^{15} k_{\mathrm{obs}} \cong 1.03-1.04$ [6]. The observed small KIE is inconsistent with the findings from the PIX experiments that indicate decomposition of $\mathrm{T}^{-}$ is partially rate-determining, which would yield a larger KIE. However, it would be very unfavorable for the $-\mathrm{NH}_{2}$ to leave with a developing negative charge. It is then possible that formation of a new $\mathrm{N}-\mathrm{H}$ bond to the solvent occurs during decomposition of $\mathrm{T}^{-}$. If formation of the $\mathrm{N}-\mathrm{H}$ bond yielded an inverse contribution to the observed KIE, the overall ${ }^{15} k_{\text {obs }}$ would be smaller than expected, as is observed.

Solvent KIEs involving hydrogen atoms have been in the literature for a very long time. These KIEs reflect the role of water as a proton donor/acceptor on going to the transition state. On the other hand, solvent nucleophile KIEs are dramatically less numerous. The mechanistic information obtained from these KIEs is both important and unique. The major drawback is that the experimental protocols are extremely difficult to 
develop. The procedure used in our laboratories $[20,22,23,24]$ for the amides, oxoesters and thioesters of formic acid is highlighted in this review to give the reader a feeling for both the complexity of measuring this type of heavy-atom KIE and the rewards from obtaining such data.

The overall hydrolysis of the amides, oxoesters, and thioesters of formic acid yields either formic acid or formate ion, depending on $\mathrm{pH}$. When formic acid is the product there are further challenges (see section 2.2). In the present case, the alkaline hydrolysis of formamide, the product is formate. Formate contains one oxygen atom coming from the original carbonyl-O of the substrate (the measured isotopic composition is called ${ }^{18} \delta_{\mathrm{C}=\mathrm{O}}$ ) and a second oxygen coming from the solvent (called ${ }^{18} \delta_{\mathrm{Nu}}$ ). ${ }^{18} \delta_{\mathrm{Nu}}$ is different than ${ }^{18} \delta$ of bulk solvent because it has been subjected to the KIE. This is shown in Scheme 3a where the filled-in circle $(\mathbf{O})$ represents the oxygen of the original carbonyl and the normal script " $\mathrm{O}$ " represents the oxygen from the solvent.
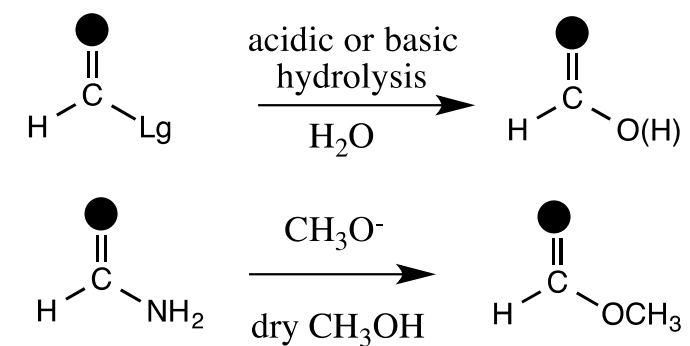

(formamide)
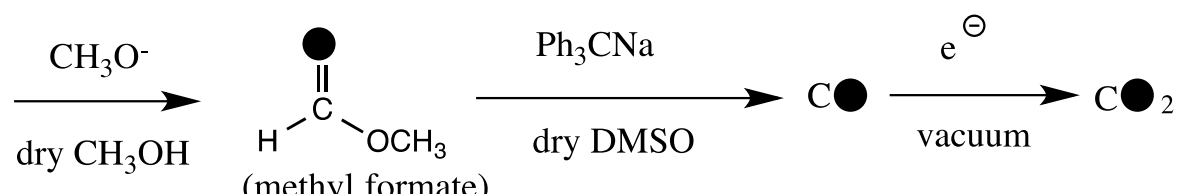<smiles>O=CCl</smiles>
1) Hydrolysis
2) $\mathrm{pH}>6$<smiles>O=CO</smiles>

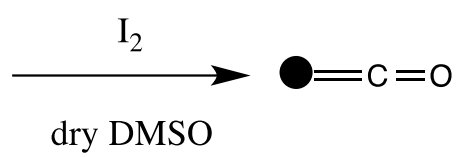

Scheme 3. Chemical reactions utilized in determination of the nucleophile-O KIE.

What is needed is a method of separately determining ${ }^{18} \delta_{\mathrm{C}=\mathrm{O}}$ and ${ }^{18} \delta_{\mathrm{Nu}}$ after complete alkaline hydrolysis of the substrate to formate. This is accomplished by the following experimental steps: (a) The ${ }^{18} \delta_{\mathrm{C}=\mathrm{O}}$ is determined from the starting amide, oxoester, or thioester. Amides require initial quantitative conversion to methyl formate. Methyl formate can be quantitatively decarbonylated to $\mathrm{CO}$, followed by conversion of $\mathrm{CO}$ to $\mathrm{CO}_{2}$ by electrolysis (Scheme $3 \mathrm{~b}$ ). The original carbonyl-O of the substrate is preserved in this process. Note: thioesters can be decarbonylated directly without conversion to methyl formate. The $\mathrm{CO}_{2}$ is isolated and ${ }^{18} \delta_{\mathrm{C}=\mathrm{O}}$ is determined by IRMS. (b) The ${ }^{18} \delta_{\mathrm{Nu}}$ is determined indirectly by quantitatively hydrolyzing the substrate to formate. In a quantitative conversion the ${ }^{18} \delta_{\mathrm{C}=\mathrm{O}}$ does not change. Next, the formate is isolated, dried under high vacuum, and oxidized to $\mathrm{CO}_{2}$ under non-aqueous conditions (Scheme $3 c)$. Finally, the $\mathrm{CO}_{2}$ is analyzed by IRMS. The measured delta $\left({ }^{18} \delta_{\text {obs }}\right)$ is the weighted average ${ }^{18} \delta$ for both of the oxygen atoms in the $\mathrm{CO}_{2}$. Since ${ }^{18} \delta_{\mathrm{C}=\mathrm{O}}$ and ${ }^{18} \delta_{\text {obs }}$ are now known, ${ }^{18} \delta_{\mathrm{Nu}}$ can be calculated via eq 3 .

$$
18 \text { obs }=(0.5)\left(\begin{array}{ll}
18 & \\
& \mathrm{C}=\mathrm{O}
\end{array}\right)+(0.5)\left(\begin{array}{ll}
18 & \mathrm{Nu}
\end{array}\right)
$$


Once the ${ }^{18} \delta_{\mathrm{Nu}}$ in formate is known, the ${ }^{18} \delta$ for the unreacted solvent nucleophile must be determined. In alkaline conditions there are two possible nucleophiles, water (probably hydrogen bonded to hydroxide) and the hydroxide ion itself. One can easily measure the ${ }^{18} \delta$ for both possible solvent nucleophiles in solution (called ${ }^{18} \delta_{\mathrm{H} 2 \mathrm{O}}$ and ${ }^{18} \delta_{\mathrm{OH}}$, respectively) using simple published procedures [25]. Because the nucleophiles are in very large excess, ${ }^{18} \delta_{\mathrm{H} 2 \mathrm{O}}$ and ${ }^{18} \delta_{\mathrm{OH}}$ do not change significantly during the reaction. As a result, this experiment yields two possible solvent nucleophile KIEs. The KIE is obtained by simply subtracting ${ }^{18} \delta_{\mathrm{H} 2 \mathrm{O}}$ or ${ }^{18} \delta_{\mathrm{OH}}$ from ${ }^{18} \delta_{\mathrm{Nu}}$. This simple subtraction works because ${ }^{18} \delta_{\mathrm{Nu}}$ was obtained after $100 \%$ hydrolysis (where ${ }^{18} \delta_{\mathrm{C}=\mathrm{O}}$ is the same in both substrate and product). Using the present case of the alkaline hydrolysis of formamide [20] as an example, if ${ }^{18} \delta_{\mathrm{H} 2 \mathrm{O}}$ is -39.6 and ${ }^{18} \delta_{\mathrm{Nu}}$ in formate is -62.3 , then the solvent nucleophile-O KIE becomes (-62.3)-(-39.6)=-22.7, which is the fractionation factor between water and the nucleophilic oxygen atom that is incorporated into formate. This means that formate becomes depleted in ${ }^{18} \mathrm{O}$ relative to water by 22 per mil, leading to a normal KIE of ${ }^{18} k_{\mathrm{obs}}=1.022$ for water as the nucleophile and hydroxide acting as a general base. A similar calculation using ${ }^{18} \delta_{\mathrm{OH}}=-79.6$ gives an inverse KIE of ${ }^{18} k_{\mathrm{obs}}=0.982$ for hydroxide as the direct nucleophile.

Can one choose between these two possible nucleophiles? In some cases this is possible, if additional information is available. Theoretical calculations [21] show that in rate-determining formation of the tetrahedral intermediate the KIE for addition of an oxygen nucleophile will be normal for early (more $\mathrm{sp}^{2}$-like) transition states and will become inverse for later (more $\mathrm{sp}^{3}$-like) transition states. In the alkaline hydrolysis of formamide it is not possible to choose between the two possible nucleophiles because the tetrahedral intermediate partitions equally between substrate and product.

There are several major methodological complications of solvent nucleophile-O KIE experiments. First, equation 3 assumes that $50 \%$ of the oxygen atoms in formate are from the original carbonyl-O of the substrate. This may not be the case when the carbonyl-O of the substrate undergoes significant ${ }^{18} \mathrm{O}$ exchange with the solvent, resulting in a small $k_{\mathrm{h}} / k_{\mathrm{ex}}$ ratio. This is exactly what occurs in the present case of the alkaline hydrolysis of formamide, where $k_{\mathrm{h}} / k_{\mathrm{ex}}=2.5$. A series of differential equations (see supporting information in reference 20) have been published that correct for this exchange and allow determination of the true value of ${ }^{18} \delta_{\mathrm{Nu}}$. Second, the product itself might independently exchange ${ }^{18} \mathrm{O}$ with solvent. This is not a problem for the present alkaline hydrolysis, where the product is formate. However, under acidic conditions where formic acid is the product, this exchange can present a serious roadblock to determining the solvent nucleophile-O KIE. In some cases this problem is solvable as discussed in section 3.2.

\subsection{The acid-catalyzed hydrolysis of formamide}

The acid-catalyzed hydrolysis of formamide was studied using the same PIX and KIE experiments [26] as discussed above (see Fig. 1B). One significant difference is that measurement of the solvent nucleophile-O KIE was not possible. Under acidic reaction conditions the product, formic acid, exchanges its oxygen atoms with the solvent at a much faster rate than overall amide hydrolysis reaction. This exchange rapidly washes 
out the two oxygen atoms in formic acid so that an accurate correction factor is not possible.

Early kinetic studies showed that amide hydrolysis is first-order in hydronium ion and second-order in water [5,27]. Our PIX experiment gave $k_{\mathrm{h}} / k_{\mathrm{ex}}>55$. Because there is so little exchange of ${ }^{18} \mathrm{O}$ into the carbonyl-O, this means that breakdown of a symmetrical tetrahedral species to product must be rapid. In turn, this argues for rate-determining formation of a tetrahedral intermediate. The proposed mechanism is given in Scheme 4. This mechanism is a truncated form of the mechanism given in reference 26 because the exchange of ${ }^{18} \mathrm{O}$ from solvent into $\mathrm{T}_{\mathrm{N}}{ }^{+}$is so slow it can be ignored. $\mathrm{In}_{\mathrm{N}}{ }^{+}$, " $\mathrm{N}$ " stands for the + charge being on the nitrogen.

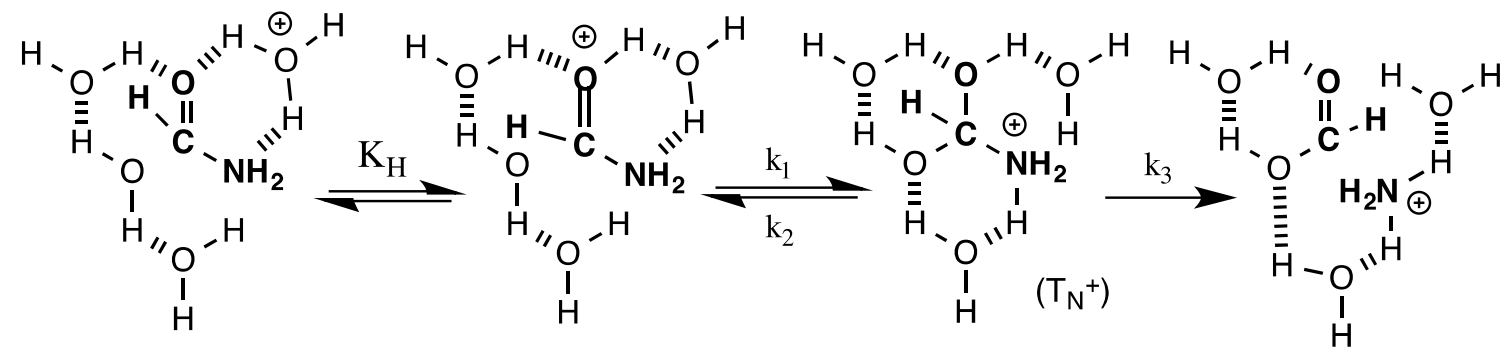

Scheme 4. Proposed mechanism for the acidic hydrolysis of formamide.

The formyl-H KIE is a large inverse effect $\left({ }^{\mathrm{D}} k_{\mathrm{obs}}=0.79\right)$ indicating the transition state contains significant $\mathrm{sp}^{3}$ character. Assuming the first step $\left(K_{\mathrm{H}}\right)$ is a rapid pre-equilibrium step and that breakdown of $\mathrm{T}_{\mathrm{N}}{ }^{+}\left(k_{3}\right)$ is also rapid, then the observed formyl-H KIE is largely derived from bonding changes on going to the transition state for the $k_{1}$ step. This transition state represented in Fig 2, Structure III, accommodates these observations. It contains a single hydronium ion and two water molecules as required by the experimentally determined rate law. In addition there is an increase in $\mathrm{sp}^{3}$ character at the carbonyl-C in this transition state, as expected on the basis of the inverse formyl-H KIE. The observed small normal leaving-N KIE $\left({ }^{15} k_{\mathrm{obs}}=1.0050\right)$ is consistent with the fact that $k_{3}$ is not significantly rate-determining and is also easily accommodated by Structure III (Fig. 2) The likely source of the normal KIE is the loss of $\pi$-bond character (from resonance) to the leaving- $\mathrm{N}$ in the transition state.

\subsection{The urease-catalyzed hydrolysis of formamide, semicarbazide and hydroxyurea}

Three alternate substrates were used in a KIE investigation of urease-catalyzed hydrolysis. The accepted mechanism in the literature involves formation of at least one tetrahedral intermediate, which precedes product release. For urea and its derivatives (semicarbazide and hydroxyurea), the initial product is a carbamic acid, which is proposed to rapidly yield $\mathrm{CO}_{2}$ (Scheme 5a). On the other hand the hydrolysis of formamide yields formate (Scheme $5 \mathrm{~b}$ ). The simplest kinetic mechanism proposed for urease-catalyzed hydrolysis of urea (as well as for these alternate substrates) is given in Scheme 5c, where T stands for tetrahedral intermediate [28]. 


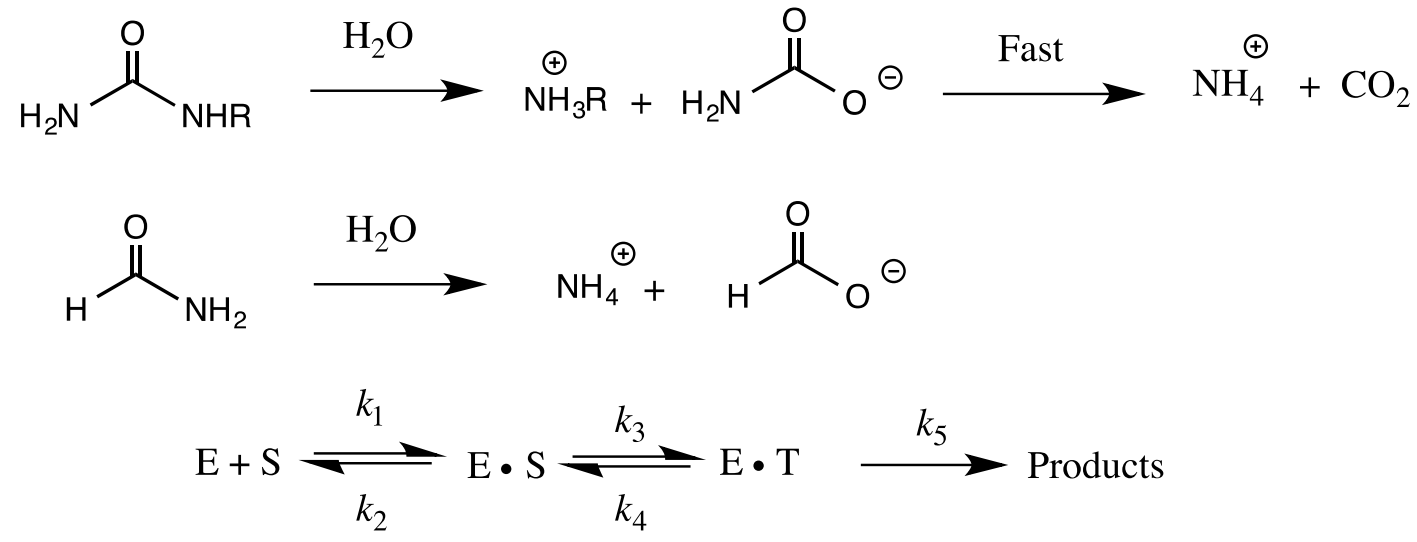

Scheme 5. Urease-catalyzed hydrolysis of (a) urea and (b) formamide. Proposed kinetic mechanism for all urease-catalyzed hydrolyses (c).

Formamide was shown to be an alternate, but slow substrate for urease [29]. Formamide hydrolysis has been subjected to the most complete KIE study (five atoms investigated, see Fig. 3A) in this series of alternative substrates [30]. The PIX experiment showed no exchange between water and formamide. This is not surprising since the chiral environment of the enzyme renders the two oxygen atoms diastereotopic and thus of intrinsically different reactivity. The formyl-H KIE for formamide hydrolysis is inverse but quite small in magnitude, where $\left({ }^{\mathrm{D}} V / K\right)_{\mathrm{obs}}=0.95$. Initially this indicates that the transition state is early ( $\mathrm{sp}^{2}$-like). However, the large observed leaving-N KIE, where $\left({ }^{15} \mathrm{~V} / \mathrm{K}\right)_{\mathrm{obs}}=1.0327$ clearly indicates that breaking the $\mathrm{C}-\mathrm{N}$ bond is mostly rate-determining and means the above initial formyl-H KIE interpretation needs to be modified. This leaving-N KIE is much larger than that published for loss of the first amino group in the natural substrate, urea, where $\left({ }^{15} V / K\right)_{\mathrm{obs}}=1.015$ [31].
A. Formamide Hydrolysis:
B. Semicarbazide Hydrolysis:

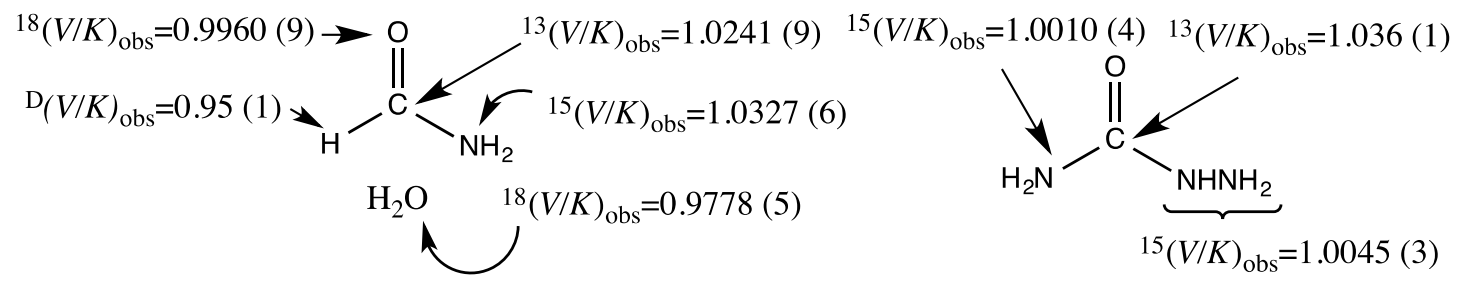

C. Hydroxyurea Hydrolysis:

D. Hydroxyurea Hydrolysis:

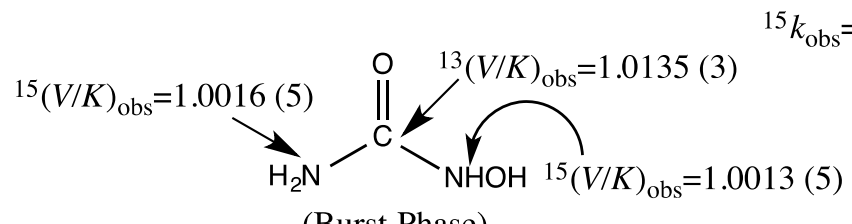

(Burst Phase)

Fig. 3: KIE Summary of Urease-Catalyzed Hydrolysis. (A) Formamide; (B) Semicarbazide; and (C and D) Hydroxyurea. Numbers in parentheses are the standard error in the right-most decimal. 
The formyl-H and leaving-N KIEs can be accommodated by the transition state structure which is given for the most widely accepted mechanism [28]. To save space only the rate-determining breakdown step of the tetrahedral intermediate $\left(k_{5}\right)$ is given (Scheme 6) where the filled in circles represent the two active site $\mathrm{Ni}$ ions required for enzyme activity; for additional mechanistic details please see reference 30 . The large leaving-N and small inverse formyl-H KIEs can be reconciled as follows. A detailed analysis of the steady state rate law leads to the conclusion that the observed formyl-H $\mathrm{KIE}$ is the product of the equilibrium isotope effect (EIE, or ${ }^{\mathrm{D}} K_{\mathrm{eq}}$ ) for formation of the tetrahedral intermediate and the KIE on the $k_{5}$ step. This is expressed mathematically as $\left({ }^{\mathrm{D}} V / K\right)_{\mathrm{obs}}=\left({ }^{\mathrm{D}} K_{\mathrm{eq}}\right) *\left({ }^{\mathrm{D}} k_{5}\right)$. The formyl-H EIE for formation of the tetrahedral intermediate was estimated earlier in section 2.1 to be ${ }^{\mathrm{D}} K_{\text {eq }} \sim 0.69$, making the intrinsic formyl-H KIE on the $k_{5}$ step equal to 1.37 [20]. This is reasonable because during this step the tetrahedral intermediate is going from $\mathrm{sp}^{3}$ hybridization to a more $\mathrm{sp}^{2}$-like hybridization in the transition state.

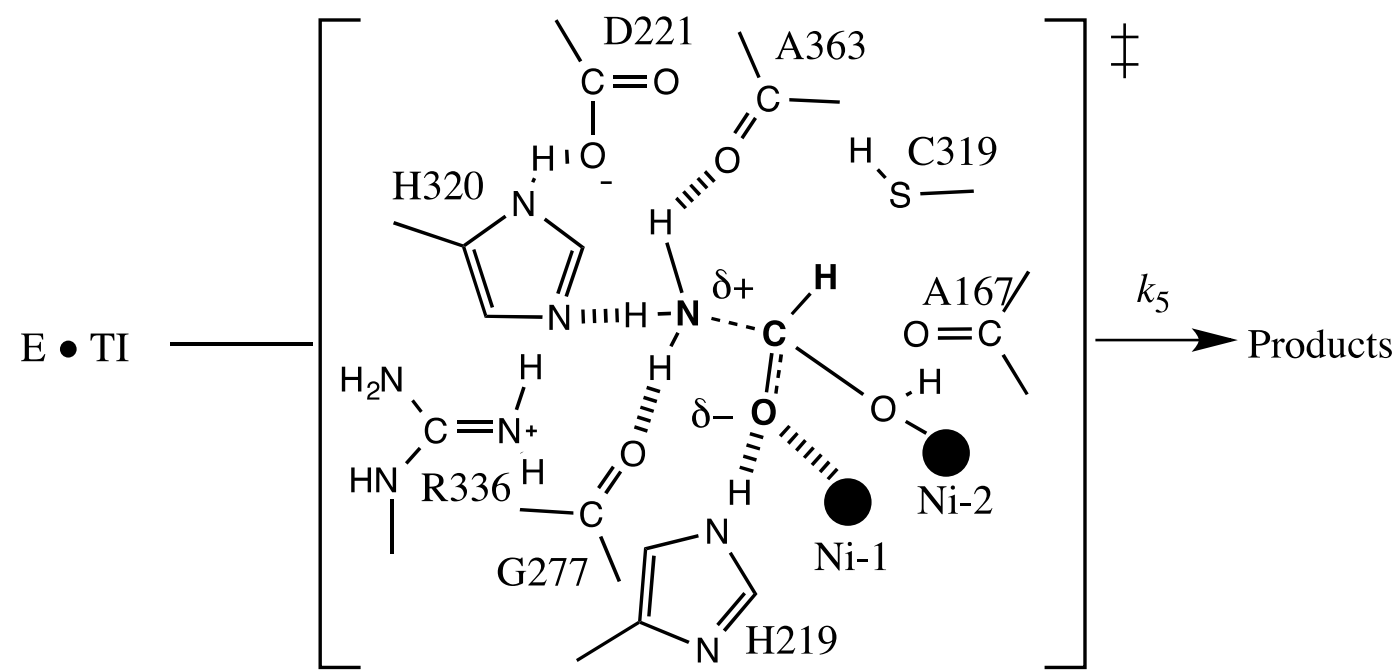

Scheme 6. Transition state for hydrolysis of formamide by urease from Jack Bean (Canavalia Ensiformis).

The solvent nucleophile-O KIE on formamide hydrolysis is large and inverse, where $\left({ }^{18} V / K\right)_{\text {obs }}=0.9778$. It is likely that bonding changes to the solvent-O in $k_{5}$ are minor compared to those in the bond formation step $\left(k_{3}\right)$ and would not greatly contribute to the observed KIE (i.e. ${ }^{18} k_{5} \sim 1.00$ ). Therefore, most of the bonding changes to the solvent oxygen atom occur during formation of the tetrahedral intermediate, making the observed effect largely an EIE. This means $\left({ }^{18} \mathrm{~V} / \mathrm{K}\right)_{\mathrm{obs}}={ }^{18} K_{\text {eq. }}$. Models for this type of equilibrium give an estimated ${ }^{18} \mathrm{O}$-EIE of approximately ${ }^{18} K_{\mathrm{eq}} \sim 0.98$, in reasonable agreement with the observed effect [30].

Semicarbazide is also known as an alternative substrate for urease [29]. Experiments to measure the KIEs on the urease-catalyzed hydrolysis of semicarbazide were specifically designed to obtain information on both of the nitrogen-containing leaving groups: $-\mathrm{NH}_{2}$ and $-\mathrm{NHNH}_{2}$ [32]. It was postulated that the first group to leave would have a larger, normal KIE because the second group would leave rapidly after irreversible release of the leaving group. Because semicarbazide is a derivative of urea, 
the product of the reaction is $\mathrm{CO}_{2}$ (Scheme 5a) and rapidly exchanges ${ }^{18} \mathrm{O}$ with water. Consequently it was not possible to measure the nucleophile-O and carbonyl-O KIEs. In addition, this substrate does not have a formyl-H, eliminating that KIE as well. As expected one of the nitrogen leaving groups gave a larger KIE than the other, where $\left({ }^{15} \mathrm{~V} / \mathrm{K}\right)_{\mathrm{obs}}=1.0045$ for $-\mathrm{NHNH}_{2}$ and $\left({ }^{15} \mathrm{~V} / \mathrm{K}\right)_{\mathrm{obs}}=1.0010$ for $-\mathrm{NH}_{2}$ (Fig. 3B). In addition, the KIE for the $-\mathrm{NHNH}_{2}$ leaving group is an average of the primary KIE of the inner nitrogen and the secondary KIE of the outer nitrogen. The secondary KIE for the outer nitrogen is expected to be very small and, as an approximation, can be largely ignored. This would then make the KIE for the inner nitrogen much closer to double the observed effect, approaching $\left({ }^{15} \mathrm{~V} / \mathrm{K}\right)_{\mathrm{obs}}=1.0090$. With this larger corrected KIE it is even clearer that $-\mathrm{NHNH}_{2}$ is the first group to leave. The smaller nitrogen $\mathrm{KIE}$ for the $-\mathrm{NHNH}_{2}$ group in semicarbazide compared to that for the $-\mathrm{NH}_{2}$ group in formamide reflects a change in mechanism, where a step prior to breakdown of the tetrahedral intermediate has become partially rate-determining. If the binding steps are rapid, the most likely candidate is formation of the tetrahedral intermediate $\left(k_{3}\right)$.

The last alternate substrate for urease to be investigated in our laboratories was hydroxyurea [33]. The KIE investigation of hydroxyurea hydrolysis was designed in a similar manner to that for semicarbazide [34]. It was hoped that hydroxyurea would offer an improvement over semicarbazide, namely elimination of the secondary KIE from the outer nitrogen of the $-\mathrm{NHNH}_{2}$ leaving group. Unlike the other alternate substrates, hydroxyurea displays a peculiar biphasic kinetic pattern. Early in the reaction there is a burst phase, followed by a later plateau phase. During the burst phase the rate of hydrolysis rapidly increases with time, whereas during the plateau phase the rate of the reaction increases very slowly (or not at all) with time. The results of the KIE investigation for the two phases were nearly identical (Fig. 3C and 3D). Therefore, it seems unlikely that there are major differences in the chemical mechanism. As a result the analysis given below will be for the plateau phase only. The two possible nitrogen leaving groups show small normal KIEs which are of similar magnitude. The KIE for the

$-\mathrm{NHOH}$ leaving group is $\left({ }^{15} \mathrm{~V} / \mathrm{K}\right)_{\mathrm{obs}}=1.0022$, while that for $-\mathrm{NH}_{2}$ is $\left({ }^{15} V / K\right)_{\mathrm{obs}}=1.0019$. As a result it is not possible to rigorously assign the order of the leaving for the two groups. What is clear is that $k_{5}$ is even less rate-determining than in the urea, formamide and semicarbazide cases.

Combining the data from the substrates studied, the leaving-N KIE steadily decreases on going from urea to semicarbazide to hydroxyurea. The commitment factors $\left(k_{5} / k_{4}\right)$ for urea and its two derivatives can be calculated from the observed KIEs plus EIEs estimated from model reactions (see reference 34 for details). In this case the larger the commitment factor the more frequently the tetrahedral intermediate goes on to products $(\mathrm{C}-\mathrm{N}$ bond breaking) versus returning to substrates. These calculated commitment factors are 1.2 for urea, 2.7 for semicarbazide and 24 for hydroxyurea. This is in line with the expectation that the better leaving group (based on $\mathrm{p} K_{\mathrm{a}}$ ) will leave first: $-\mathrm{NH}_{2}\left(\mathrm{p} K_{\mathrm{a}}=9.6\right),-\mathrm{NHNH}_{2}\left(\mathrm{p} K_{\mathrm{a}}=8.1\right)$ and $-\mathrm{NHOH}\left(\mathrm{p} K_{\mathrm{a}}=6.0\right)$. The better the leaving group, the faster the $k_{5}$ step becomes relative to the $k_{4}$ step.

\subsection{The alkaline hydrolysis of methyl formate}

The stepwise mechanism of the alkaline hydrolysis of alkyl oxoesters (Scheme 7) has been established for a long time [35]. From a KIE standpoint, the most highly studied 
alkaline hydrolysis is that for methyl formate (Fig. 4A) [22]. Published PIX experiments gave $k_{\mathrm{h}} / k_{\mathrm{ex}}=18.3$, consistent with a partition ratio of $k_{3} / k_{2}=9.2$ and consistent with ratedetermining formation of the tetrahedral intermediate (the $k_{1}$ step of Scheme 7) [36].

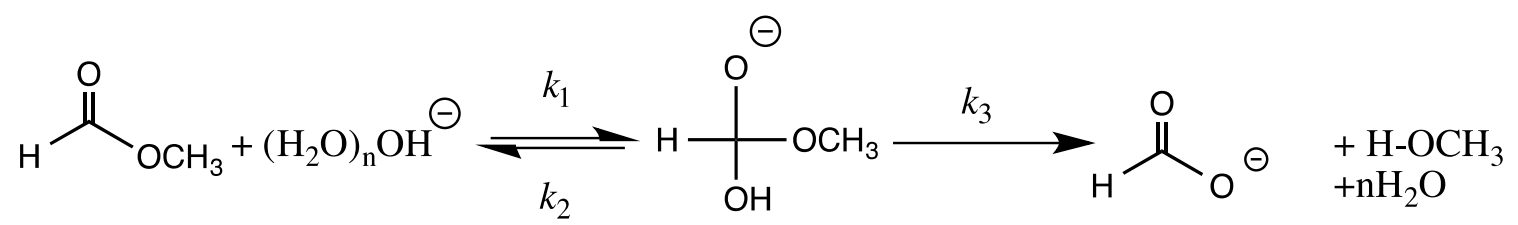

Scheme 7. Proposed mechanism for the alkaline hydrolysis of methyl formate.

The small leaving-O KIE $\left({ }^{18} k_{\mathrm{obs}}=1.009\right)$ is consistent with the above interpretation and is likely due to the loss of resonance (a partial $\pi$ bond) to the leaving-O on going to the transition state [36]. The formyl-H KIE was ${ }^{\mathrm{D}} k_{\mathrm{obs}}=0.95$ and indicates that the transition state for formation of the tetrahedral intermediate is quite early and contains little $\mathrm{sp}^{3}$ character [37].

A. Alkaline Hydrolysis:

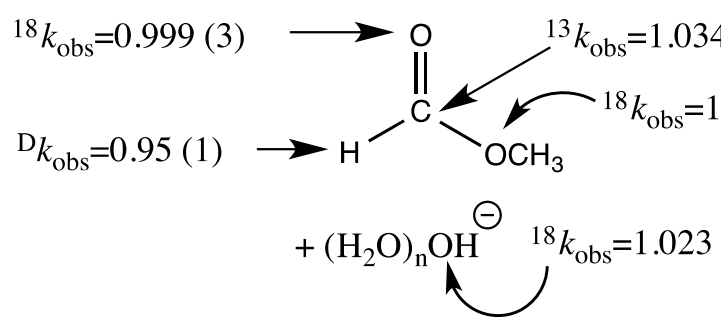

B. Acid-Catalyzed Hydrolysis:

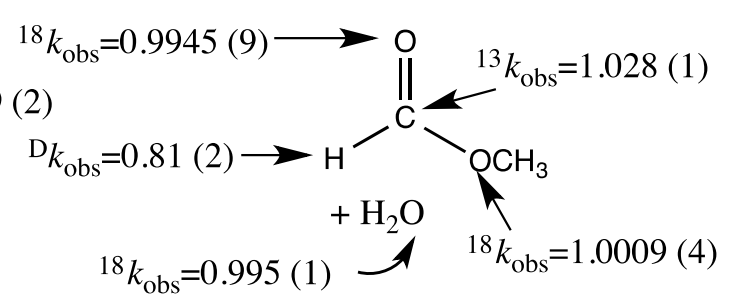

Fig. 4: KIE Summary of the Alkaline (A) and Acid-Catalyzed Hydrolysis (B) of Methyl Formate. Numbers in parentheses are the standard error in the right-most decimal.

The key experiment for this reaction was the solvent nucleophile-O KIE [22]. The general procedure for measuring this KIE was presented in section 2.1. Once again there are two possible nucleophiles from the solvent, water molecules in the hydrogen bonding sphere of hydroxide or hydroxide itself. The experiment yields the same two possible solvent nucleophile-O KIEs as in the alkaline hydrolysis of formamide, based on determination of ${ }^{18} \delta_{\mathrm{Nu}},{ }^{18} \delta_{\mathrm{OH}}$ and ${ }^{18} \delta_{\mathrm{H} 2 \mathrm{O}}$. If $\mathrm{OH}^{-}$is the direct nucleophile, then ${ }^{18} k_{\mathrm{obs}}=0.983$. If a coordinating water molecule is the direct nucleophile, then ${ }^{18} k_{\mathrm{obs}}=1.023$. Here the latter choice is the most logical one. Recall that theoretical calculations show that early transition states (with little $\mathrm{sp}^{3}$ character) occurring during formation of a tetrahedral intermediate lead to the expectation of a normal KIE [21]. Therefore, this result favors water as the actual nucleophile, with general base assistance from hydroxide. The transition state is shown below as Structure IV (Fig. 5). The conclusion that water is the actual nucleophile is also strongly supported by a proton inventory study that found at least two hydrogen atoms are involved in the transition state. This is consistent with the general base mechanism, but not the direct attack by hydroxide [38]. 


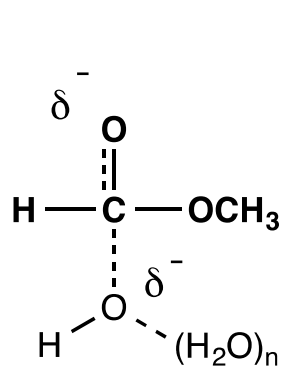

IV<smiles>CO[C@H]1O[C@H]([OH2+])O[C@H]1[OH2+]</smiles>

V

Fig. 5. Possible transition state structures for alkaline hydrolysis (IV) and acidic hydrolysis (V) of methyl formate.

\subsection{The acid-catalyzed hydrolysis of methyl formate}

The kinetics of the acid-catalyzed hydrolysis of methyl formate, like many oxoesters, is first-order in hydronium ion and is probably second-order in water [39]. The PIX experiment [36] shows a somewhat lower level of exchange $\left(k_{\mathrm{h}} / k_{\mathrm{ex}}=11.3\right)$ than initially expected. The calculated partition ratio is $k_{3} / k_{2}=5.7$, which is still consistent with a more rapid $k_{3}$ step. The PIX results indicate that formation of the tetrahedral intermediate, not its breakdown, is mostly rate-determining and the transition state of highest energy occurs during the $k_{1}$ step. The leaving-O KIE is very small and is consistent with the above interpretation [36]. The formyl-H KIE is inverse $\left({ }^{\mathrm{D}} k_{\mathrm{obs}}=0.81\right)$ and of greater magnitude than that for alkaline hydrolysis $\left({ }^{\mathrm{D}} k_{\mathrm{obs}}=0.95\right)$, consistent with a later transition state containing considerable $\mathrm{sp}^{3}$ character (Fig. 4B) [37]. The most likely mechanism to fit all these data is that shown in Scheme 8. The ${ }^{18} \mathrm{O}$ exchange pathway is not given because it is minor [23].

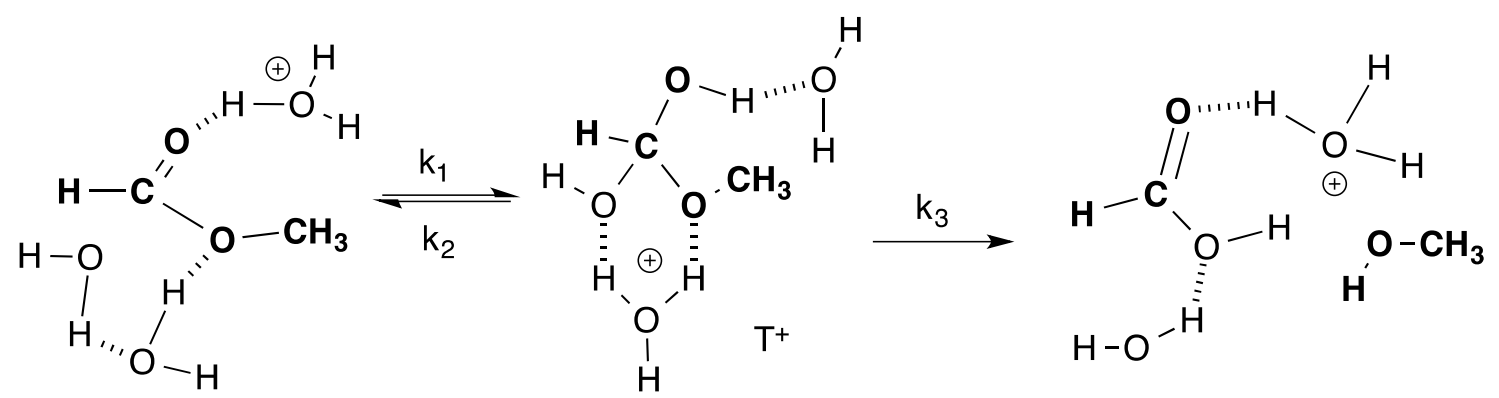

Scheme 8. Proposed mechanism for acidic hydrolysis of methyl formate.

Unlike the acid-catalyzed hydrolysis of amides, it was possible to measure the solvent nucleophile-O KIE for this case. The reason is subtle. In the case of methyl formate the acid-catalyzed hydrolysis is fast compared to the independently determined exchange of ${ }^{18} \mathrm{O}$ into formic acid. Some exchange does occur, but the amount is small enough that a minor correction factor can be applied (see supporting information of reference 23). There is no problem in the assignment of which solvent atom is the nucleophile since under acid conditions the only reasonable nucleophile present is water. The observed solvent nucleophile-O KIE is inverse but very small $\left({ }^{18} k_{\mathrm{obs}}=0.995\right)$. Since the leaving-O KIE is also so small, analysis of the observed nucleophile-O KIE requires 
estimation of the EIE $\left({ }^{18} k_{1} 1{ }^{18} k_{2}\right.$ or $\left.{ }^{18} K_{\text {eq }}\right)$ on formation of T $\mathrm{T}^{+}$. Model equilibria under acidic conditions suggest that ${ }^{18} K_{\text {eq }}$ should be about 0.968 [23]. From this estimated EIE and rate constant ratios obtained from the PIX experiment, the intrinsic KIE can be estimated to be ${ }^{18} k_{1} \sim 1.017$. It is not surprising that the intrinsic KIE on $k_{1}$ is normal. However, the magnitude of the KIE is larger than expected based on the prediction of a relatively late transition state. Theory predicts that a large inverse formyl-H KIE on tetrahedral intermediate formation should be coupled with a small normal or small inverse nucleophile-O KIE [21]. One possible reason for this discrepancy is the uncertainty in estimation of the EIE from model reactions. A more rigorous theoretical calculation of the EIE may prove helpful. The proposed transition state is given as Structure V (Fig. 5).

\subsection{Oxoesters: The chymotrypsin-catalyzed hydrolysis of specific ester substrates}

Hydrolysis of oxoesters by esterases has not been subjected to the multiple heavyatom KIE studies discussed above for the non-enzymatic hydrolyses. Two examples are given here. The leaving-O KIE has been determined for a few specific substrates of chymotrypsin, two of which are: methyl $N$-acetyl-L-tryptophan, where ${ }^{18}(\mathrm{~V} / \mathrm{K})_{\mathrm{obs}}=1.007$ [40], and ethyl $N$-acetyl-L-tryptophan, where ${ }^{18}(V / K)_{\mathrm{obs}}=1.0117$ [41]. These results indicate that expulsion of the leaving group is at least partially rate-determining.

\subsection{Thioesters: The alkaline hydrolysis of formylthiocholine (FTC)}

Bruice and others utilized kinetics and solvent hydrogen KIEs to investigate nonenzymatic thioester hydrolysis in acidic, neutral and basic conditions $[42,43,44,45]$. In each case the results of these experiments pointed to a stepwise mechanism with the formation of tetrahedral intermediates. The $\mathrm{pH}$-rate profile for alkyl thioformates displays catalysis by hydroxide under alkaline conditions. This catalysis appears to be first-order in $\left[\mathrm{OH}^{-}\right]$. Alkaline conditions are difficult to investigate because the reaction rate is so much faster than under acidic and neutral conditions. Nevertheless we have developed techniques in our laboratories to obtain results from PIX and KIE experiments [24]. The substrate used was formylthiocholine (FTC), which is shown in Fig. 6. FTC was also chosen because it is a substrate for butyrylcholinesterase (BChE)-catalyzed hydrolysis and allows for a comparison of the enzymatic reaction to those without enzyme. 
A. Alkaline Hydrolysis:

$$
\begin{gathered}
\mathrm{D}_{\mathrm{obs}}=0.88(2) \\
\text { C. Neutral Hydrolysis: }
\end{gathered}
$$

$$
{ }^{\mathrm{D}} k_{\mathrm{obs}}=0.75(2){ }_{+}^{\mathrm{H}_{2} \mathrm{O} \longleftarrow}{ }^{18} k_{\mathrm{obs}}=0.9917(9)
$$

B. Acid-Catalyzed Hydrolysis:

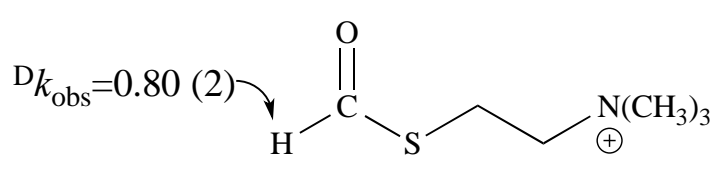

Fig. 6. KIE Summary of the alkaline (A), acid-catalyzed (B), neutral (C) and BChEcatalyzed (D) hydrolysis of FTC. Numbers in parentheses are the standard error in the right-most decimal.

First, a ${ }^{13} \mathrm{C}-\mathrm{NMR}$ method was developed to measure the exchange of ${ }^{18} \mathrm{O}$ from water into one of the oxygen atoms of the product. This method relied on accurate integration of the ${ }^{18} \mathrm{O}$-shifted ${ }^{13} \mathrm{C}$-carbonyl resonances of formate. Measuring exchange into product is only viable as long as the product itself does not independently undergo ${ }^{18} \mathrm{O}$ exchange, as is the case under alkaline conditions. Since the reaction is rapid it was run in a limiting amount of $\mathrm{OH}^{-}$so that all the FTC would not be consumed. The results of these PIX experiments were unequivocal: There was no detectible exchange of ${ }^{18} \mathrm{O}$ into the carbonyl-O of FTC within the analytical limits of the method $\left(k_{\mathrm{h}} / k_{\mathrm{ex}}>25\right)$, implying that breakdown of a tetrahedral intermediate to products is not rate-determining. The accepted mechanism for alkaline hydrolysis of alkyl thioesters such as FTC mirrors that for oxoesters and is shown in Scheme 9. It is interesting to note here that all the hydrolysis conditions investigated (alkaline, acidic, neutral and enzyme-catalyzed) resulted the same lack of exchange within the limits of the technique, consistent with a rapid $k_{3}$ step. This is not a complete surprise because sulfur is an excellent leaving group.

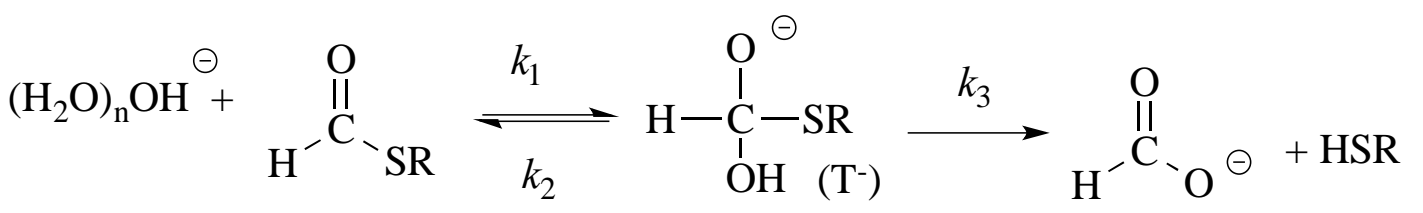

Scheme 9. Proposed mechanism for the alkaline hydrolysis of FTC.

The formyl-H, and the solvent nucleophile-O KIEs (Fig. 6A) proved most important in the development of a workable transition state structure for alkaline hydrolysis. The formyl-H KIE is inverse and somewhat small $\left({ }^{\mathrm{D}} k_{\mathrm{obs}}=0.88\right)$, similar to that found in the alkaline hydrolysis of methyl formate $\left({ }^{\mathrm{D}} k_{\mathrm{obs}}=0.95\right)$. This result indicates a 
relatively early transition state (containing considerable $\mathrm{sp}^{2}$ character), which occurs during formation of the tetrahedral intermediate. The solvent nucleophile-O KIE is ${ }^{18} k_{\mathrm{obs}}=1.029$ for a water nucleophile and ${ }^{18} k_{\mathrm{obs}}=0.989$ for a hydroxide nucleophile. The interpretation of these results is similar to that for alkaline hydrolysis of methyl formate. By theory, normal solvent nucleophile KIEs are expected when there is an early transition state for formation of a tetrahedral intermediate [21]. Therefore, the best choice of the actual solvent nucleophile is water, with hydroxide acting as a general base (Fig 7., Structure VI). However, the formyl-H KIE is a larger inverse effect $\left({ }^{\mathrm{D}} k_{\mathrm{obs}}=0.88\right)$ for FTC than for methyl formate $\left({ }^{\mathrm{D}} k_{\mathrm{obs}}=0.95\right)$. This result together with the fact that the reaction has both an excellent nucleophile and an excellent leaving group requires consideration of the concerted mechanism as an alternative. The transition state for a concerted mechanism (Structure VII) would also fit the KIE results. Concerted mechanisms have been observed in acyl group transfers involving very good nucleophiles and good leaving groups such as $p$-nitrophenyl [15].
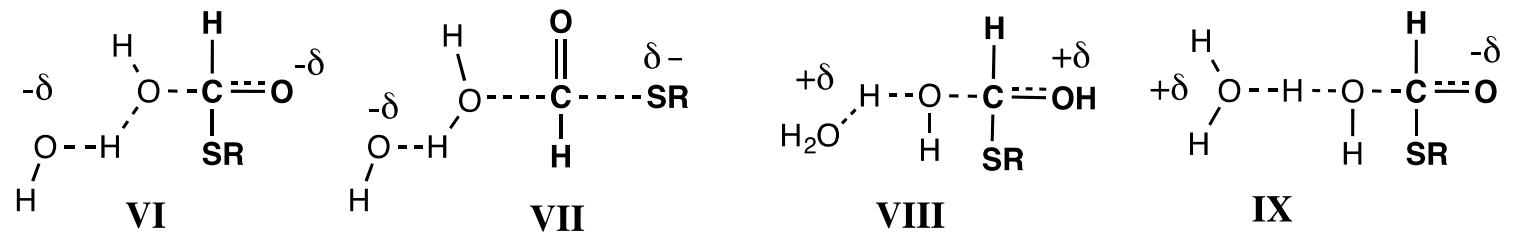

Fig 7. Possible transition state structures for the alkaline (VI and VII), acidic (VIII) and neutral (IX) hydrolysis of FTC.

The leaving-S KIE offers a solution to this dilemma. Since there is no detectable ${ }^{18} \mathrm{O}$ exchange, the stepwise mechanism should show a leaving-S KIE near unity because breakdown of the tetrahedral intermediate, which involves $\mathrm{C}-\mathrm{S}$ bond breaking, occurs after the rate-determining step. In the concerted mechanism there would be some $\mathrm{C}-\mathrm{S}$ bond-breaking. In turn, this should lead to the observation of a normal sulfur KIE. The semi-classical limit of a KIE for $\mathrm{C}-\mathrm{S}$ bond cleavage is approximately 1.015-1.017, well within the precision of our methodology [6]. Work on developing a procedure to measure the leaving-S KIE is in progress in our laboratories.

\subsection{The acid-catalyzed hydrolysis of FTC}

The acid-catalyzed hydrolysis of alkyl thioesters like FTC has been shown to be first order in hydrogen ion concentration [44]. In the PIX experiment FTC displayed no detectable ${ }^{18} \mathrm{O}$ exchange within experimental limits [24]. In this particular experiment only exchange into FTC (not formic acid) can be used because of the known ${ }^{18} \mathrm{O}$ exchange into formic acid. The working hypothesis from these results is that the stepwise mechanism is operating as proposed for other thioesters and that formation of the tetrahedral intermediate is rate-determining (Scheme 10). It should be noted that acylactivated thioesters, like trifluorothioacetate, show a decreasing rate of hydrolysis as a function of increasing hydronium ion concentration below $\mathrm{pH} 2[42,43]$. These types of thioesters are not the subject of this review. 


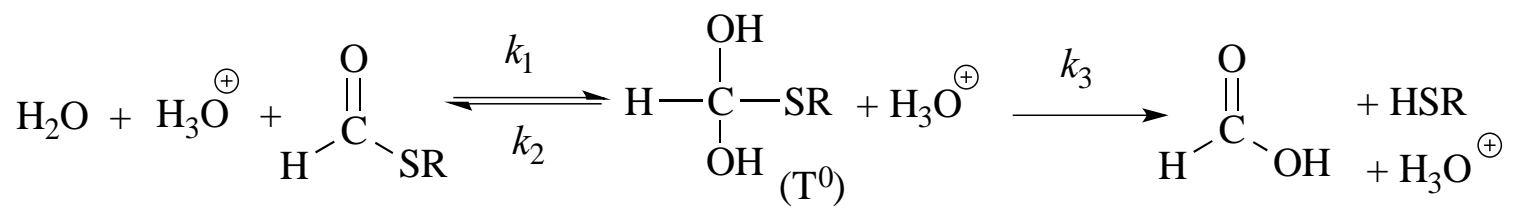

Scheme 10. Proposed mechanism for acidic hydrolysis of FTC.

The formyl-H KIE is a moderately large inverse effect $\left({ }^{\mathrm{D}} k_{\mathrm{obs}}=0.80\right)$, indicating more $\mathrm{sp}^{3}$ character in the transition state than in the alkaline hydrolysis case (Fig. 6B). The proposed transition state based on the above results is shown in Structure VIII (Fig. 7). Because ${ }^{18} \mathrm{O}$ from the solvent exchanges into formic acid much faster than overall hydrolysis, it was not possible to measure the solvent nucleophile-O KIE. As in the alkaline hydrolysis case, leaving-S KIEs should verify that there is very little $\mathrm{C}-\mathrm{S}$ bond breaking in the transition state.

\subsection{The neutral hydrolysis of FTC}

Thioesters are reactive enough to undergo an observable hydrolysis under neutral conditions [44]. In this region of the $\mathrm{pH}$-rate profile $(\mathrm{pH} \mathrm{2-7)}$ the reaction rate is roughly independent of $\mathrm{pH}$. The proposed mechanism is again stepwise (Scheme 11). As in the alkaline and acidic hydrolysis cases, no ${ }^{18} \mathrm{O}$ exchange was detectable within the limits of our PIX methodology. Therefore, formation of the tetrahedral intermediate appears to be mostly rate-determining. The formyl-H KIE (Fig. 6C) is even more inverse $\left(k_{\mathrm{obs}}=0.75\right)$ than in the acid-catalyzed hydrolysis case, indicating even more $\mathrm{sp}^{3}$ character in the transition state [21].

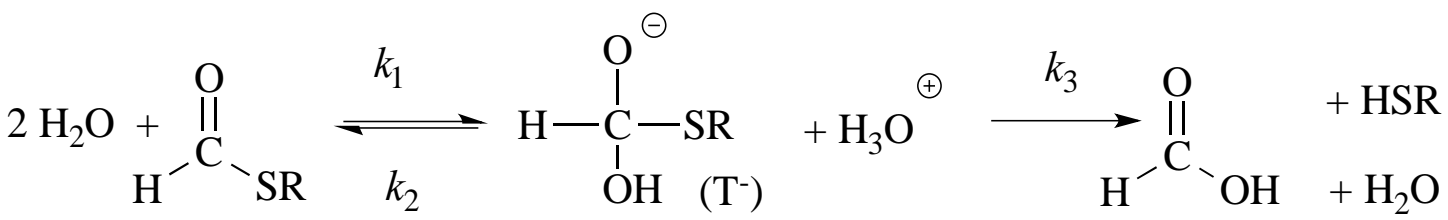

Scheme 11. Proposed mechanism for the neutral hydrolysis of FTC.

In this case it was possible to measure the solvent nucleophile-O KIE because the addition of a small amount of buffer kept the $\mathrm{pH}$ greater than 5.0 during the reaction. This ensured that the product would be formate, which does not exchange ${ }^{18} \mathrm{O}$ with the

solvent. The solvent nucleophile-O KIE was small and inverse $\left({ }^{18} k_{\mathrm{obs}}=0.9917\right)$. Unlike the alkaline hydrolysis case, water is the only nucleophile possible. The most likely explanation is that this KIE arises from both reaction coordinate motion (TIF) and stiffer bonds being formed in the transition state (TDF). Since the former is always normal and the later can be inverse (as in this case), the observed KIE appears to be dominated slightly by the TDF factor [21]. The proposed transition state is shown as structure IX, Fig. 7. Measurement of the leaving-S KIE is in progress.

\subsection{The Butyrylcholinesterase (BChE)-catalyzed hydrolysis of FTC}


Both acetylthiocholine and FTC are alternate substrates for BChE. The products of hydrolysis are thiocholine plus either acetate or formate. The kinetic mechanism is ping-pong, with formation of an acyl enzyme being the "ping" and hydrolysis of the acyl enzyme being the "pong" (see Scheme 12) [46]. Since water is the only substrate added in the second half of the reaction, this step has typically been difficult to study.

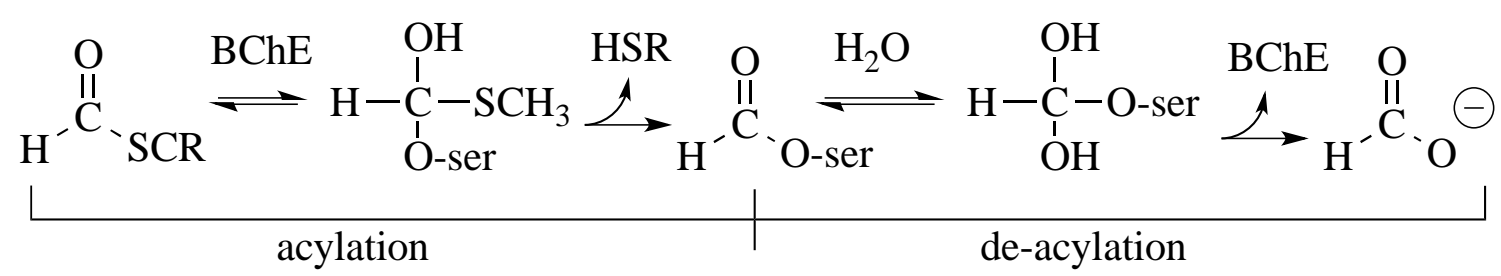

Scheme 12. The ping-pong mechanism for hydrolysis of FTC by butyrylcholinesterase.

The PIX experiment did not detect ${ }^{18} \mathrm{O}$ exchange from solvent into the carbonyl-O of FTC [24]. Once again this is not surprising because the chiral environment of the enzyme renders the two oxygen atoms diastereotopic and thus of intrinsically different reactivity. The formyl-H KIE (Fig. 6D) was measured by the competitive method. Such KIEs can give information about bonding changes up to and including the first irreversible step. This means that the observed KIE reflects bonding changes on going to the highest energy transition state of the acylation step, prior to the irreversible release of thiocholine. The formyl-H KIE is small and inverse, where ${ }^{\mathrm{D}}(V / \mathrm{K})_{\mathrm{obs}}=0.89$, like that for alkaline hydrolysis of FTC, where ${ }^{\mathrm{D}}(V / \mathrm{K})_{\mathrm{obs}}=0.88$. This result indicates that the transition state has only a small amount of $\mathrm{sp}^{3}$ character. Published $\beta$-hydrogen KIEs for acetylthiocholine are in agreement with this conclusion [46]. Because of the ambiguity of the PIX results, these KIE data do not distinguish between a transition state that occurs during formation of the tetrahedral intermediate and one that occurs during its decomposition. Work in progress on the leaving-S KIE can make this distinction. Up to this point the existing data is consistent with a transition state like that for alkaline hydrolysis (Fig 7, Structure VI), except in this case the nucleophile is a serine hydroxyl group.

In contrast to the formyl-H KIE, the solvent nucleophile-O KIE gives information only on de-acylation (the "pong" step), because water is only involved in this step. The observed solvent nucleophile-O KIE is ${ }^{18}(\mathrm{~V} / \mathrm{K})_{\text {obs }}=0.9925$, very similar to that for the neutral hydrolysis of FTC, where ${ }^{18} k_{\mathrm{obs}}=0.9917$. Once again there are two possible interpretations. First, breakdown of the acyl enzyme might be rate-determining, where the leaving group is a serine hydroxyl. If this were the case, the oxygen atom in the tetrahedral intermediate that came from water would be in equilibrium with the oxygen atom of solvent water molecules. Changes in bonding to the nucleophilic-O are expected to be much smaller during the decomposition of the tetrahedral intermediate than during its formation, because the decomposition occurs after the new bond to the nucleophile has been formed. With this assumption the observed KIE would largely be an EIE on formation of the tetrahedral intermediate. Based on known model reactions this EIE should be quite large and inverse $\left({ }^{18} K_{\mathrm{obs}}<0.98\right)$, like that observed in the urease-catalyzed hydrolysis of formamide, where ${ }^{18}(V / K)_{\mathrm{obs}}=0.9778$ [30]. Clearly this is not the case. The second and more likely possibility is that the rate-determining step(s) occur prior to 
breakdown of the tetrahedral intermediate to products. For example, this could be similar to neutral hydrolysis, where the observed KIE arises during formation of the tetrahedral intermediate. The observed small inverse KIE is the result of the inverse contribution from the TDF being slightly larger than the normal contribution from the TDF.

\subsection{General conclusions and mechanistic commonalities}

A combination of PIX and KIE experiments from our laboratories has helped refine and extend previous mechanistic work on the hydrolysis of amides, oxoesters, and thioesters. Since KIEs disturb reaction mechanisms less than other physical organic techniques, the experiments themselves are unlikely to change the natural mechanism. For many years the accepted mechanism for most acyl group hydrolyses has been the stepwise mechanism with formation of various tetrahedral intermediates. In fact this is generally the only mechanism presented in sophomore-level organic chemistry textbooks. There are a few cases [15] where there is evidence for the concerted pathway (Scheme 1b). It was thought that alkaline hydrolysis of FTC had the best chance of reacting via this mechanism because of the combination of a very good leaving group and strong nucleophile. The leaving-S KIE, which is in progress, may be able to distinguish between these two mechanisms. The following sections summarize how the results of PIX and KIEs helped establish commonalities (and differences) of the stepwise mechanisms for various hydrolyses studied.

\subsection{Determining if formation or breakdown of a tetrahedral intermediate is rate- determining}

Most of the non-enzymatic reaction mechanisms studied in our laboratories are thought to contain at least one kinetically significant tetrahedral intermediate. Information on whether formation or breakdown of the tetrahedral species is ratedetermining can often be inferred from PIX experiments in combination with leaving

group KIEs. A small amount of ${ }^{18} \mathrm{O}$ exchange (i.e. large $k_{\mathrm{h}} / k_{\mathrm{ex}}$ ratios) and a small leaving group KIE are strong evidence for some step prior decomposition of tetrahedral intermediate to products is rate-determining. In these cases the likely rate-determining step is formation of the tetrahedral intermediate. Large amounts of ${ }^{18} \mathrm{O}$ exchange (i.e. $k_{\mathrm{h}} / k_{\text {ex }}$ ratios near 1.0 or lower) and a large leaving group KIE are strong evidence for ratedetermining decomposition of a tetrahedral intermediate.

Under alkaline conditions the hydrolysis of oxoesters and thioesters exhibit large $k_{\mathrm{h}} / k_{\text {ex }}$ ratios and small leaving group KIEs, implicating formation of a tetrahedral intermediate as the rate-determining step. The exception is the alkaline hydrolysis of formamide under low concentrations of hydroxide, where $k_{\mathrm{h}} / k_{\mathrm{ex}}=2.5$. Graphical extrapolation of a plot of $k_{\mathrm{h}} / k_{\mathrm{ex}} \mathrm{Vs}$. [OH$\left.{ }^{-}\right]$leads to a partition ratio of $k_{3} / k_{2}=1.05$ [20]. This infers similar rates of formation and breakdown of the tetrahedral intermediate and further infers transition states for the $k_{3}$ and $k_{1}$ steps are of roughly equal energy. The smaller than expected leaving-N KIE is attributed to the fact the nitrogen is unlikely to leave with a large developing negative charge. The formation of a new $\mathrm{N}-\mathrm{H}$ bond results in the smaller KIE.

Under acidic conditions the PIX experiments indicate very small amounts of ${ }^{18} \mathrm{O}$ exchange $\left(k_{\mathrm{h}} / k_{\mathrm{ex}}>25\right)$. The one exception is the acid-catalyzed hydrolysis of methyl 
formate, where $k_{\mathrm{h}} / k_{\mathrm{ex}}=11.3$. However, even in this case breakdown of the tetrahedral intermediate to products is still favored $\left(k_{3} / k_{2}=5.6\right)$. The leaving group KIEs are all very small, in agreement with the conclusion that formation of the tetrahedral intermediate is rate-determining.

In the $\mathrm{BChE}$ and urease catalyzed reactions no exchange of ${ }^{18} \mathrm{O}$ into the substrate carbonyl-O was observed. However, this is likely due to the fact that enzyme active sites are chiral and can distinguish between the two oxygen atoms of the tetrahedral species. For this reason it is not possible to rely on the PIX experiment to ascertain whether formation or breakdown of a tetrahedral intermediate is rate-determining. However, the leaving group KIE can often provide this information. In the case of the urease-catalyzed hydrolysis of formamide, the leaving-N KIE is very large $\left[{ }^{15}(\mathrm{~V} / \mathrm{K})_{\mathrm{obs}}=1.0327\right]$ and is consistent with rate-determining breakdown of the last tetrahedral intermediate to ammonium formate. The leaving-N KIEs for the urease-catalyzed hydrolysis of urea $\left[{ }^{15}(\mathrm{~V} / \mathrm{K})_{\mathrm{obs}}=1.0150\right]$, semicarbazide $\left[{ }^{15}(\mathrm{~V} / \mathrm{K})_{\mathrm{obs}}=1.090\right]$ and hydroxyurea $\left[{ }^{15}(\mathrm{~V} / \mathrm{K})_{\mathrm{obs}}=1.0022\right]$ indicate formation of a tetrahedral species is becoming progressively more rate-determining.

\subsection{Determination of the $\mathrm{sp}^{3}$ character of the tetrahedral intermediate}

Once it is known from the PIX experiment and the leaving group KIEs whether formation or breakdown of the tetrahedral intermediate is rate-determining, the formyl-H KIE has the potential to estimate how much $\mathrm{sp}^{3}$ character is present in the transition state. In cases where formation of the tetrahedral intermediate is rate-determining a large inverse formyl-H KIE $\left({ }^{\mathrm{D}} k_{\mathrm{obs}}=0.75-0.80\right)$ indicates a relatively late, $\mathrm{sp}^{3}$-like transition state, while a small inverse $\mathrm{KIE}\left({ }^{\mathrm{D}} k_{\mathrm{obs}}=0.88-0.95\right)$ argues in favor of an earlier sp ${ }^{2}$-like transition state. These qualitative conclusions are consistent with theoretical calculations $[21]$.

These conclusions do not hold when breakdown of the tetrahedral intermediate is either partially (alkaline hydrolysis of formamide) or fully rate-determining (ureasecatalyzed hydrolysis of formamide). In the former case one must consider the observed formyl-H KIE as a complex product of the KIEs for both formation and decomposition of the tetrahedral intermediate. In the latter case the observed KIE is the product of the EIE on formation and the KIE on breakdown of the tetrahedral intermediate to products.

\subsection{Discoveries from the solvent nucleophile KIE}

Solvent nucleophile KIEs are very difficult to measure but provide important additional information about transition state structure that is not available from other types of KIEs. Under alkaline conditions there are two possible nucleophiles, a water molecule hydrogen-bonded to hydroxide or hydroxide ion itself. Because the EIE between water and hydroxide is known, it is possible to identify the actual nucleophile in some cases. If the formation of the tetrahedral intermediate is rate-determining and the formyl-H KIE is small and inverse, theoretical calculations indicate that the observed solvent nucleophile-O KIE should be normal. In the alkaline hydrolysis of methyl formate and FTC this is exactly what is observed. In these cases a strong argument can be made for water being the direct nucleophile with general base assistance from hydroxide (sections 2.1 and 3.1). In other cases with a large inverse formyl-H KIE it is not possible to make a rigorous assignment of the actual nucleophile. For alkaline hydrolysis of 
formamide the nearly equal energy of the transition states for formation and decomposition of the tetrahedral intermediate makes the assignment of the actual nucleophile difficult.

\subsection{Why have the carbonyl-C and carbonyl-O KIEs been difficult to interpret?}

The theoretical calculations of carbonyl-O and carbonyl-C KIEs mentioned above are quite old [21], but indicate that the magnitude of the carbonyl-C KIE decreases slowly as this carbon atom moves across the reaction coordinate. Empirical results on the hydrolysis of aryl carbonates support this hypothesis [47]. It is hoped that theoreticians will revisit this topic. For this reason the observed carbonyl-C KIEs are listed in this review even though there were not utilized in determination of transition state bonding.

The carbonyl-O KIE has also been difficult to interpret, but for different reasons. This KIE is technically a secondary KIE because the connection between the carbonyl-O and the carbonyl-C is not completely severed during the reaction. Nevertheless, several bonding changes do occur simultaneously during the reaction in solution. First, $\pi$-bond order is being reduced (a normal contribution to the KIE). Second, new torsional and bending modes are being formed (an inverse contribution to the KIE). Third, the carbonyl-O is undergoing a change in bonding to hydrogen atoms of the solvent (normal or inverse). This last issue has been raised recently in the literature. Cox has argued that in many cases tetrahedral intermediates with full positive or negative charge on an oxygen atom are generally too unstable to exist in aqueous solution [17]. Instead he proposes that charge is spread out through a chain of solvent water molecules (via the Grotthuss mechanism). Once again it is hoped that the carbonyl-O KIE will eventually be studied more thoroughly from a theoretical framework.

\subsection{Future directions.}

The reaction of amides, oxoesters, and thioesters are very important in both chemistry and biology. Considerable empirical information concerning the nonenzymatic and enzyme-catalyzed mechanisms of these groups has been published over the years. This review presents the results from stable isotope experiments, which have deepened our understanding of the rate-determining step(s) and the structure of the transition states for the hydrolysis reaction. Certainly more empirical data on different types of acyl groups and nucleophiles is warranted. However, it is hoped that the large quantity of empirical data presented in this review will stimulate high-level theoretical studies of the more poorly understood aspects of these KIEs.

\section{Acknowledgements}

We posthumously thank professor W. W. Cleland (University of Wisconsin-Madison) for use of his laboratory and his isotope ratio mass spectrometer from 1990-2014. We also thank him for sharing his thoughtful insights on our work and for his immense contributions to the study of enzyme mechanisms. Research in our laboratories was supported in the past by State Faculty Support Grants (Cal Poly) to J.F.M., Cottrell College Science award from Research Corporation to JFM and is currently supported by a grant from the National Science Foundation (CHE-1049689). 


\section{References}

[1] S.L. Johnson, General Base and Nucleophilic Catalysis of Ester Hydrolysis and Related Reactions, Adv. Phys. Org. Chem. 5 (1967) 237-330.

[2] E.A. Castro, Kinetics and Mechanisms of Reactions of Thiol, Thiono, and Dithio Analogues of Carboxylic Esters with Nucleophiles, Chem Rev 99 (1999) 3505-3524.

[3] J.F. Marlier, Multiple Isotope Effects on the Acyl Group Transfer Reactions of Amides and Esters, Acc. Chem. Res. 34 (2001) 283-290.

[4] A.C. Hengge, Isotope Effects in the Study of Phosphoryl and Sulfuryl Transfer Reactions, Acc Chem Res 35 (2002) 105-112.

[5] R.S. Brown, A.J. Bennet, H. Slebocka-Tilk, Recent Perspectives Concerning the Mechanism of $\mathrm{H}_{3} \mathrm{O}^{+}$and Hydroxide-Promoted Amide Hydrolysis, Acc. Chem. Res 23 (2002) 481-488.

[6] W.P. Huskey, Origins and Interpretation of Heavy-Atom Isotope Effects., in: P.F. Cook (Ed.), Enzyme Mechanisms from Isotope Effects, CRC Press, Boca Raton, FL, 1991, pp. 38-72.

[7] L. Melander, W.H. Saunders, Reaction Rates of Isotopic Molecules, Wiley, New York, 1980.

[8] K.C. Westaway, Determining Transition State Structure Using Kinetic Isotope Effects, J. Label. Compd. Radiopharm. 50 (2007) 989-1005.

[9] A.C. Hengge, Isotope Effects in Chemistry and Biology, in: A. Kohen, H.-H. Limbach (Eds.), Secondary Isotope Effects, Taylor \& Francis, Boca Raton, 2006.

[10] D.B. Northrop, Determining the Absolute Magnitude of Hydrogen Isotope Effects, Isotope Effects on Enzyme-Catalyzed Reactions, Cleland, W. W. O’Leary, M.H.Northrop, D.B., University Park Press 1977, pp. 122-152.

[11] M.L. Bender, H.d.A. Heck, Carbonyl Oxygen Exchange in General Base-Catalyzed Ester Hydrolysis, J. Am. Chem. Soc. 89 (1967) 1211-1220.

[12] S. Basaif, A.K. Luthra, A. Williams, Concerted Acetyl Group Transfer between Substituted Phenolate Ion Nucleophiles - Variation of Transition-State Structure as a Function of Substituent, J. Am. Chem. Soc. 111 (1989) 26472652.

[13] J.P. Guthrie, Concerted Mechanism for Alcoholysis of Esters: An Examination of the Requirements, J. Am. Chem. Soc. 113 (1991) 3941-3949.

[14] D. Stefanidis, W.P. Jencks, General Base Catalysis of Ester Hydrolysis, J. Am. Chem. Soc. 115 (1993) 6045-6050.

[15] A.C. Hengge, R.A. Hess, Concerted or Stepwise Mechanisms for Acyl TransferReactions of P-Nitrophenyl Acetate - Transition-State Structures from Isotope Effects, J. Am. Chem. Soc. 116 (1994) 11256-11263.

[16] T.W. Bentley, D.N. Ebdon, E.J. Kim, I.S. Koo, Solvent Polarity and Organic Reactivity in Mixed Solvents: Evidence Using a Reactive Molecular Probe to Assess the Role of Preferential Solvation in Aqueous Alcohols, J. Org. Chem. 70 (2005) 1647-1653. 
[17] R.A. Cox, A Greatly under-Appreciated Fundamental Principle of Physical Organic Chemistry, Int. J. Mol. Sci. 12 (2011) 8316-8332.

[18] S.S. Biechler, R.W. Taft, The Effect of Structure on Kinetics and Mechanism of the Alkaline Hydrolysis of Anilides, J. Am. Chem. Soc. 79 (1957) 4927-4935.

[19] R.L. Schowen, H. Jayaraman, L. Kershner, Catalytic Efficiencies in Amide Hydrolysis. The Two-Step Mechanism, J Am Chem Soc 88 (1966) 3373-3375.

[20] J.F. Marlier, N.C. Dopke, K.R. Johnstone, T.J. Wirdzig, A Heavy-Atom Isotope Effect Study of the Hydrolysis of Formamide, J. Am. Chem. Soc. 121 (1999) 4356-4363.

[21] J.L. Hogg, R. J., I. Kovach, R.L. Schowen, Kinetic Isotope-Effect Probes of Transition-State Structure. Vibrational Analysis of Model Transition States for Carbonyl Addition, J. Am. Chem. Soc. 102 (1980) 79-85.

[22] J.F. Marlier, Heavy-Atom Isotope Effects on the Alkaline Hydrolysis of Methyl Formate: The Role of Hydroxide Ion in Ester Hydrolysis, J. Am. Chem. Soc. 115 (1993) 5953-5956.

[23] J.F. Marlier, T.G. Frey, J.A. Mallory, W.W. Cleland, Multiple Isotope Effect Study of the Acid-Catalyzed Hydrolysis of Methyl Formate, J. Org. Chem 70 (2005) 1737-1744.

[24] L.I. Robins, K.M. Meisenheimer, E.J. Fogle, C.A. Chaplan, R.L. Redman, J.T. Vacca, M.R. Tellier, B.R. Collins, D.H. Duong, K. Schulz, J.F. Marlier, A Kinetic Isotope Effect and Isotope Exchange Study of the Nonenzymatic and the Equine Serum Butyrylcholinesterase-Catalyzed Thioester Hydrolysis, J. Org. Chem. 78 (2013) 12029-12039.

[25] M. Green, H. Taube, Isotopic Fractionation in the Oh--H2o Exchange Reaction, J. Phys. Chem. 67 (1963) 1565-1566.

[26] J.F. Marlier, E. Campbell, C. Lai, M. Weber, L.A. Reinhardt, W.W. Cleland, Multiple Isotope Effect Study of the Acid-Catalyzed Hydrolysis of Formamide, J. Org. Chem. 71 (2006) 3829-3836.

[27] V.K. Krieble, K.A. Holst, Amide Hydrolysis with High Concentrations of Mineral Acids, J. Am. Chem. Soc. 60 (1938) 2976-2980.

[28] P.A. Karplus, M.A. Pearson, R.P. Hausinger, 70 Years of Crystalline Urease: What Have We Learned?, Acc. Chem. Res. 30 (1997) 330-337.

[29] C. Gazzola, R.L. Blakeley, B. Zerner, On the Substrate Specificity of Jack Bean Urease (Urea Amidohydrolase, Ec 3.5.1.5), Can. J. Biochem. 51 (1973) 13251330.

[30] J.F. Marlier, W.W. Cleland, Multiple Isotope Effect Study of the Hydrolysis of Formamide by Urease from Jack Bean (Canavalia Ensiformis), Biochemistry 45 (2006) 9940-9948.

[31] H.L. Schmidt, Isotope Effect on Each, C- and N-Atoms, as a Tool for the Elucidation of Enzyme-Catalyzed Amide Hydrolysis, in: H.L. Schmidt, H. Forstel, K. Heinzinger (Eds.), Stable Isotopes, Analytical Chemistry Symposia Series, Elsevier Scientific Publishing Company, Amsterdam-Oxford-New York, 1982, pp. 77-82.

[32] J.F. Marlier, E.J. Fogle, W.W. Cleland, A Heavy-Atom Isotope Effect and Kinetic Investigation of the Hydrolysis of Semicarbazide by Urease from Jack Bean (Canavalia Ensiformis), Biochemistry 47 (2008) 11158-11163. 
[33] W.N. Fishbein, T.S. Winter, J.D. Davidson, Urease Catalysis .I. Stoichiometry Specificity and Kinetics of a Second Substrate - Hydroxyurea, J. Biol. Chem. 240 (1965) 2402-2406.

[34] J.F. Marlier, L.I. Robins, K.A. Tucker, J. Rawlings, M.A. Anderson, W.W. Cleland, A Kinetic and Isotope Effect Investigation of the Urease-Catalyzed Hydrolysis of Hydroxyurea, Biochemistry 49 (2010) 8213-8219.

[35] M.L. Bender, Mechanisms of Catalysis of Nucleophilic Reactions of Carboxylic Acid Derivatives, Chem. Rev. 60 (1960) 53-113.

[36] C.B. Sawyer, J.F. Kirsch, Kinetic Isotope-Effects for Reactions of Methyl FormateMethoxyl-0-18 J. Am. Chem. Soc. 95 (1973) 7375-7381.

[37] Z. Bilkadi, R.D. Lorimier, J.F. Kirsch, Secondary Alpha-Deuterium Kinetic Isotope-Effects and Transition-State Structures for Hydrolysis and Hydrazinolysis Reactions of Formate Esters, J. Am. Chem. Soc. 97 (1975) 4317-4322.

[38] J.F. Mata-Segreda, Hydroxide as General Base in the Saponification of Ethyl Acetate, J. Am. Chem. Soc. 124 (2002) 2259-2262.

[39] K. Yates, Kinetics of Ester Hydrolysis in Concentrated Acid, Acc. Chem. Res. 4 (1971) 136-144.

[40] M.H. O'Leary, Enzyme Reaction Mechanisms from Heavy-Atom Isotope Effects, in: W.W. Cleland, M.H. O'Leary, D.B. Northrop (Eds.), Isotope Effects on Enzyme-Catalyzed Reactions, University Park Press, Baltimore (Md.), 1977, pp. 233-251.

[41] C.B. Sawyer, J.F. Kirsch, Kinetic Isotope Effects for the Chymotrypsin Catalyzed Hydrolysis of Ethoxyl-18 0 Labeled Specific Ester Substrates., J. Am. Chem. Soc. 97 (1975) 1963-1964.

[42] T.C. Bruice, L.R. Fedor, Nucleophilic Displacement Reactions at the Thiolester Bond. Iii. Kinetic Demonstrations of Metastable Intermediates in the Hydroxylaminolysis and Methoxylaminolysis of Thiolesters and Thilactones in Aqueous Solutions. , J. Am. Chem. Soc. 86 (1964) 4886-4897.

[43] L.R. Fedor, T.C. Bruice, Nucleophilic Displacement Reactions at the Thiol Ester Bond. Iv. General Base Catalyzed Hydrolysis of Ethyl Trifluorothiolacetate. Kinetic Evidence for the Formation of Tetrahedral Intermediate. , J. Am. Chem. Soc. 87 (1965) 4138-4147.

[44] R. Hershfield, G.L. Schmir, Mechanism of the Acid-Catalyzed Hydrolysis of Methyl Thiolformate, J. Am. Chem. Soc. 94 (1972) 1263-1270.

[45] R. Hershfield, G.L. Schmir, Hydrolysis of Acyl-Activated Thiol Esters. Acid Catalysis and Acid Inhibition, J. Am. Chem. Soc. 95 (1973) 3994-4002.

[46] J.R. Tormos, K.L. Wiley, Y. Wang, D. Fournier, P. Masson, F. Nachon, D.M. Quinn, Accumulation of Tetrahedral Intermediates in Cholinesterase Catalysis: A Secondary Isotope Effect Study, J. Am. Chem. Soc. 132 (2010) 17751-17759.

[47] J.F. Marlier, M.H. O'Leary, Carbon Kinetic Isotope Effects on the Hydrolysis of Aryl Carbonates, J. Am. Chem. Soc. 112 (1990) 5996-5998. 


\section{Graphical Abstract}

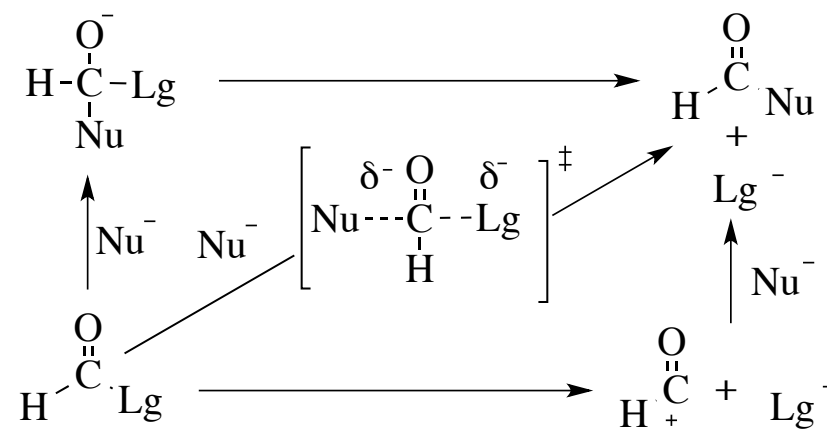

$$
(\mathrm{Nu}=\text { nucleophile; } \mathrm{Lg}=\text { leaving group })
$$

